\title{
Une étude sur l'estimation non linéaire basée sur la théorie des observateurs invariants
}

\section{A Study of non linear state estimation based on invariant observers}

\author{
Jean-Philippe Condomines ${ }^{1}$ \\ ${ }^{1}$ Laboratoire ENAC, Ecole Nationale de l'Aviation Civile, Toulouse, France, jean-philippe.condomines@enac.fr
}

RÉSUMÉ. L'objet du présent article vise à analyser une solution algorithmique d'estimation non linéaire récemment développée : l'IUKF (Invariant Unscented Kalman Filter). A l'instar des travaux menés il y a quelques années autour de l'IEKF (Invariant Extended Kalman Filter), les gains de correction de cet estimateur, construits pour être invariant, peuvent être obtenus en suivant les étapes de calcul propres au filtrage de type UKF (que celles-ci soient déclinées dans une forme factorisée ou non). Toutefois, l'intégration à la théorie des observateurs invariants d'une procédure de calcul des gains de correction qui suit un schéma algorithmique emprunté au filtrage de Kalman dit unscented, nécessite un certain nombre de développements méthodologiques. L'auteur commençe donc cet article par une recherche bibliographique. Elle présente succinctement les développements méthodologiques permettant l'élaboration des algorithmes UKF et EKF sous une forme invariante, sur la base de la théorie des observateurs invariants. Ces deux algorithmes sont ensuite exploités sur différents problèmes et comparés à leur forme standard.

ABSTRACT. This article presents a study of non linear state estimation based on invariant preserving observer : I'IUKF (Invariant Unscented Kalman Filter). Several research works on nonlinear invariant observers have been led and provide a geometrical-based constructive method for designing filters such as the IEKF (Invariant Extended Kalman Filter) wihle preserving the physical properties and systems symmetries. The developed IUKF estimator suggests a systematic approach to determine all the symmetry-preserving correction terms, without requiring any linearization of differential equations or compatibility condition. The IEKF and IUKF algorithms are compared on academic case of tilt sensor system. The developed IUKF is then used with an aided Inertial Navigation System (INS). The results show promising perpectives and demonstrate that nonlinear state estimation converges on a much bigger set of trajectories than for more traditional approaches.

MOTS-CLÉS. Estimation d'état non linéaire, filtre de Kalman sigma-point invariant, fitre de kalman étendu invariant, invariance et symétrie, système de navigation.

KEYWORDS. Nonlinear state estimation, invariant unscented Kalman filter, invariant extended Kalman filter, navigation system.

\section{Introduction}

De nombreux travaux de recherche sur les observateurs invariants non linéaires ont été menés durant ces dix dernières années notamment par P. Rouchon, S. Bonnabel et E. Salaun dont les résultats ont permis d'élaborer une méthode constructive utilisant conjointement la géométrie différentielle et la théorie des groupes pour synthétiser des filtres non linéaires dédiés aux problèmes d'estimation non linéaire de l'état d'un système. Cette dernière décénnie se caractérise donc par l'essor de nouvelles techniques, remettant en cause la définition classique de l'erreur d'estimation d'état utilisée traditionnellement dans les méthodes de conception d'observateur non linéaire. Les premiers travaux basés sur une approche géométrique sont le fruit de (Maithripala et al. [2005],Mahony et al. [2008],Lagemann et al. [2008],Aghannan et al. [2002]). Ils exploitent principalement les groupes géométriques de Lie (quaternion, matrice de rotation, etc.). Prenant ses racines dans les travaux de Krener et Isidori, elle vise à utiliser l'invariance d'un système non linéaire vis-à-vis de certaines transformations géométriques (rotation, translation, etc) pour construire un observateur ayant une convergence bien meilleure que localement autour de tout point d'équilibre (Bourmaud and al. [2013],Bourmaud and al. [2014], Khosravian et al. [2015]). Les nombreux 
résultats obtenus par Bonnabel (Bonnabel [2007], Bonnabel et al. [2008],Bonnabel et al. [2009],Bonnabel et al. [2009]) ont ainsi permis de proposer une théorie des observateurs invariants pour une classe importante de systèmes qui présentent des symétries. Désormais, la linéarisation ne se fera plus autour d'un point d'équilibre mais autour de l'élément neutre d'un groupe, ce qui permettra, comme nous le verrons, d'élargir le domaine de convergence de l'estimation et d'avoir une forme simplifiée de la dynamique de l'erreur d'estimation sur l'état. L'émergence de nouvelles applications dans le domaine de la robotique mobile et aérienne a favorisé l'essor de cette approche bien adaptée pour le problème d'estimation d'attitude (Crassidis et al. [2007],Martin et al. [2007],Martin et al. [2008],Vasconcelos et al [2008],Barczyk et al. [2013]). Ainsi, dans [Bonnabel et al., 2009], l'erreur invariante d'estimation est utilisée dans le schéma récursif du filtre de Kalman étendu pour élargir a priori son domaine de convergence. Cette hybridation aboutit à un filtre de Kalman étendu invariant (IEKF) avec de nombreuses variantes (Barrau et al. [2013],Barrau et al. [2014],Barrau et al. [2015],Barczyk et al. [2015],Li et al. [2015]).

A l'instar des travaux menés il y a quelques années autour de l'IEKF (Invariant Extended Kalman Filter), les gains de correction de cet estimateur, construit pour être invariant, peuvent êtres obtenus en suivant les étapes de calcul propres au filtrage de type UKF (Van der Merwer et al. [2001],Julier [2002],Sarkka [2007]). Toutefois, l'intégration à la théorie des observateurs invariants d'une procédure de calcul des gains de correction qui suit un schéma algorithmique emprunté au filtrage de Kalman dit unscented, nécessite un certain nombre de développements méthodologiques. Ces travaux de recherche ont conduit in fine à la mise au point de deux variantes algorithmiques de l'UKF baptisées $\pi$-IUKF (Condomines et al. [2013], Condomines et al. [2014]), utilisant une condition de compatibilité $\pi$ et IUKF (pour Invariant Unscented Kalman Filter) (Condomines and al. [2017]), sans condition de compatibilité. Les recherches menées autour des formulations des filtres $\pi$-(SR)IUKF et (SR)IUKF, ont notamment permis de montrer qu'il était donc possible, à partir des principes de base du filtrage de Kalman par Sigma Points (SPKF), de synthétiser des filtres invariants pour l'estimation d'état de systèmes dynamiques nonlinéaires caractérisés eux aussi par un domaine de convergence élargi.

Par ailleurs, le fait de recourir à un calcul des gains qui se fonde sur une technique de type UKF présente le grand avantage de ne pas nécessiter de linéarisation explicite de la dynamique de l'erreur d'état invariante, qui représente en soi un point dur de l'approche IEKF, pour identifier les matrices $\mathbf{A}(I)$ et $\mathbf{C}(I)$ (qui dépendent des invariants du problème) nécessaires à l'application des principes du filtrage de Kalman étendu. Bien qu'une différenciation analytique de cette dynamique peut être obtenue dans certains cas particuliers de modélisation (essentiellement lorsque les mouvements du système obéissent à des relations cinématiques), levant ainsi la difficulté mentionnée précédemment, les développements théoriques des filtres (SR)IUKF et $\pi$-(SR)IUKF se justifient par le fait qu'ils visent à être largement appliqués, dans le futur, à tout type de système dynamique. Cette ambition couvre notamment le cas où le modèle de la dynamique du système ne permet plus, du fait de son niveau de complexité intrinsèque, de procéder à cette linéarisation de manière analytique. Nous pensons ici notamment au cas des minidrones à voilure fixe pour lesquels des représentations numériques, sous la forme d'abaques tabulées par exemple, décrivant l'aérodynamique et la propulsion de tels engins, peuvent être avantageusement (car elles représentent une forte connaissance a priori du système) utilisées pour décrire de façon plus complète la dynamique du vol de ces appareils. A travers les différentes définitions qui suivent, nous présentons ici le fil conducteur des principaux travaux de recherche menés autour de la synthèse de filtres 
invariants. Cette théorie sera appliquée pour le développement de l'IEKF et de l'IUKF, qui a l'avantage de ne pas utiliser de condition de compatibilité, puis repris au travers de l'élaboration de deux systèmes nommés tilt sensor system et INS (Inertial Navigation System).

Le présent article comprend quatre sections. La première partie $(\$ 1)$ a trait au développement de principes méthodologiques originaux qui ont permis d'élaborer deux algorithmes d'estimation non linéaire : l'IEKF et l'IUKF. Ceux-ci fondés conjointement sur la théorie des observateurs invariants et sur les principes du filtrage de Kalman dit unscented, permettent d'apporter une solution algorithmique extrêmement intéressante aux problèmes de la convergence autour de toute trajectoire permanente. Nous avons donc mené, dans la deuxième partie $(\$ 2)$ de cet article, une étude comparative entre l'IEKF et l'IUKF sur un exemple de motivation nommé tilt sensor system. Enfin la dernière partie $(\$ 3, \S 4)$ fournit un ensemble complet de résultats pour l'IUKF avec une comparaison des performances des algorithmes SRUKF (standard) et IUKF. Cette analyse est effectuée sur la base de données simulées bruitées d'un modèle d'INS (Inertial Navigation System) qui intègrent les imperfections des différents capteurs. Ces résultats valident l'approche qui permet de déterminer une correction spécifique de la prédiction fournie par n'importe quelle représentation d'état non linéaire utilisée pour l'estimation, de sorte que la dynamique de l'observateur synthétisé vérifie aussi les propriétés de symétrie du système considéré.

\section{Notations mathématiques}

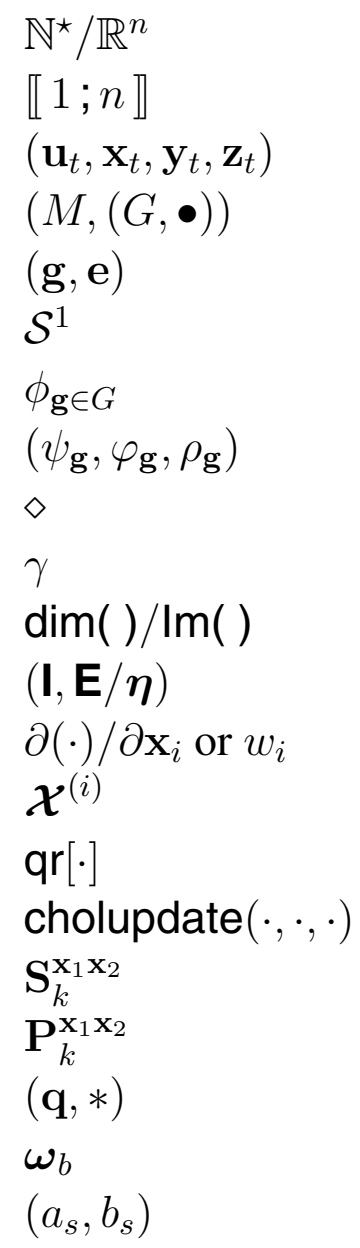

Ensemble des entiers naturels/espace Euclidien de dimension $n$ Entiers naturels $\geq 1$ et $\leq n$

Entrées, états, sorties et vecteur de mesures au temps $t$ Variété différentiable, goupe de Lie

Groupe strandard et élément neutre

Variété du cercle

Groupe de transformation

Actions locales sur les entrées, les états et les sorties

Loi de composition de groupe

Repère mobile

Dimension de l'espace des vecteurs / fonction image

Invariants fondamentaux, erreurs invariantes de sorties/ d'états

Base Euclidienne

$i^{\text {th }}$ sigma point

Factorisation QR

Décomposition de cholesky

Racine carrée de la matrice de covariance à l'instant $k$

Matrice des erreurs de covariances estimées entre $\left(\mathbf{x}_{1}, \mathbf{x}_{2}\right)$

Quaternion s.t. $\mathbf{q}=\left(q_{1} q_{2} q_{3} q_{4}\right)^{T}$, produit Hamiltonien

Vecteur correspondant aux biais sur les gyromètres

Facteur d'échelle sur les mesures des accéléromètres et magnétomètres 


\section{Petit tour d'horizon des problèmes d'estimation des filtres de Kalman invariants}

\subsection{Théorie des observateurs invariants}

Tout d'abord, considérons la représentation d'état non linéaire suivante définie par :

$$
\frac{d \mathbf{x}}{d t}=f(\mathbf{x}, \mathbf{u}), \mathbf{y}=h(\mathbf{x}, \mathbf{u})
$$

Dans l'équation [1], le vecteur d'état $\mathbf{x}$ (resp. d'entrée $\mathbf{u}$ )(resp. de sortie $\mathbf{y})$ appartient à un ensemble ouvert $\mathcal{X} \in \mathbb{R}^{n}$ (resp. $\mathcal{U} \in \mathbb{R}^{m}$ )(resp. $\mathcal{Y} \in \mathbb{R}^{p}, p \leq n$ ). Etant donné cette représentation non linéaire, nous commençons par définir une structure de groupe sur laquelle nous allons construire différentes transformations dont les actions sur les ouverts précédemment définis vont laisser inchanger la dynamique (du système).

Definition 1.1. Carte différentiable et groupe de Lie : Une variété topologique $M$ de dimension r est dite différentiable si et seulement si ses cartes de transition sont différentiables. Ainsi un groupe de Lie G est une variété différentiable qui est munie d'une structure algébrique de groupe.

Definition 1.2. Groupe de transformation : Soit $G$ un groupe de Lie et $\mathcal{T}$ un ouvert de $\mathbb{R}^{q}$. Une action sur $\mathcal{T}$ d'un groupe de transformation paramétré par $G$, noté $\left(\theta_{g}\right)_{g \in G}$ et d'application identité $\theta_{e}(e$, est l'élément neutre de $G)$, et tel que $(g, \xi) \in G \times \mathcal{T} \mapsto \theta_{g}(\xi) \in \mathcal{T}$, est une carte différentiable qui vérifie :

$-\forall \xi \in \mathcal{T}, \theta_{e}(\xi)=\xi$

- $\forall\left(g_{1}, g_{2}\right) \in G^{2}, \theta_{g_{2}} \circ \theta_{g_{1}}(\xi)=\theta_{g_{2} g_{1}}(\xi)$.

Definition 1.3. Identification du groupe de Lie et de l'ouvert : Soit $\left(\theta_{g}\right)_{g \in G}$ un groupe de transformation de rang plein i.e., $\forall g \in G$, $\operatorname{dim}\left(\operatorname{Im}\left(\theta_{g}\right)\right)=\operatorname{dim}(G)=r . \operatorname{Si} \operatorname{dim}(G)=r=\operatorname{dim}(\mathcal{T})=q$ alors $G$ et $\mathcal{T}$ peuvent être identifiés l'un à l'autre de sorte que l'application $\theta$ paramétrisée sur G puisse s'assimiler à une multiplication à droite ou à gauche telle que :

$$
\forall(g, \xi) \in(G \times \mathcal{T}), \theta_{g}(\xi)=\xi g^{-1}=R_{g^{-1}}(\xi) \quad \text { ou } \quad \theta_{g}(\xi)=g \xi=L_{g}(\xi)
$$

A partir de la définition 2, il en résulte que $\left(\theta_{g}\right)_{g \in G}$ est un difféomorphisme. Par ailleurs, nous considérerons uniquement dans la suite les actions de groupe de rang plein pour lesquelles $\operatorname{rg}\left(\theta_{g}\right)=\operatorname{dim}(G)$ et $\operatorname{dim}(G)=\operatorname{dim}(M)$. Dans ce cas là, la définition 2 signifie que nous pouvons identifier le groupe $G$ à l'ouvert $\mathcal{T}$. Par analogie, en considérant la représentation d'état [1] où le vecteur d'état (resp. d'entrée)(resp. de sortie) appartient à un ensemble ouvert $\mathcal{X} \in \mathbb{R}^{n}$ (resp. $\left.\mathcal{U} \in \mathbb{R}^{m}\right)\left(\right.$ resp. $\left.\mathcal{Y} \in \mathbb{R}^{p}, p \leq n\right)$, nous considérons le groupe de transformation défini par une multiplication à droite sur $\mathcal{X} \times \mathcal{U} \times \mathcal{Y}$ tel que :

$$
\begin{aligned}
\phi: G \times(\mathcal{X} \times \mathcal{U} \times \mathcal{Y}) & \rightarrow(\mathcal{X} \times \mathcal{U} \times \mathcal{Y}) \\
(g, \mathbf{x}, \mathbf{u}, \mathbf{y}) & \mapsto \phi_{g}(\mathbf{x}, \mathbf{u}, \mathbf{y})=\left(\varphi_{g}(\mathbf{x}), \psi_{g}(\mathbf{u}), \rho_{g}(\mathbf{y})\right)=(\mathbf{X}, \mathbf{U}, \mathbf{Y})
\end{aligned}
$$

où $\left(\varphi_{g}, \psi_{g}, \rho_{g}\right)$ sont trois difféomorphismes paramétrisés par $g \in G$ où le groupe de Lie $G$ vérifie $\operatorname{dim}(G)$ $=\operatorname{dim}(\mathcal{X})=n$. Les coordonnées des transformations ci-dessus doivent être définies telle que leur action respective mais conjuguée sur les variables d'état, d'entrée et de sortie laisse l'entière dynamique du système inchangée, c'est à dire $\dot{\mathbf{X}}=f(\mathbf{X}, \mathbf{U})$ and $\mathbf{Y}=h(\mathbf{X}, \mathbf{U})$ tel que : 
Definition 1.4. Système G-invariant et sortie équivariante : La dynamique du système sera dite Ginvariante si $\exists\left(\varphi_{g}, \psi_{g}\right)_{g \in G}, \forall(g, \mathbf{x}, \mathbf{u}) \in G \times(\mathcal{X} \times \mathcal{U})$ :

$$
f\left(\varphi_{g}(\mathbf{x}), \psi_{g}(\mathbf{u})\right)=D \varphi_{g}(\mathbf{x}) \cdot f(\mathbf{x}, \mathbf{u})
$$

où $D \varphi_{g}(\mathbf{x})$ désigne l'application dérivée de $\varphi_{g}$ en $\mathbf{x}$. De plus, sa sortie sera dite G-equivariante si $\exists\left(\rho_{g}\right)_{g \in G} G \times \mathbf{y} \mapsto \mathbf{y}, \forall(g, \mathbf{x}, \mathbf{u}) \in G \times(\mathcal{X} \times \mathcal{U})$ :

$$
h\left(\varphi_{g}(\mathbf{x}), \psi_{g}(\mathbf{u})\right)=\rho_{g}(h(\mathbf{x}, \mathbf{u}))
$$

La dernière définition signifie que les équations d'évolution et d'observation restent explicitement identiques à la suite de l'application des trois transformations. Basé sur la méthode du repère mobile de Cartan, un ensemble complet de $n$-invariant(s) dans $G$ peut être construit en considérant seulement l'action de groupe $\psi_{g}$. Prenons par exemple le cas particulier des systèmes linéaires stationnaires :

$$
\frac{d \mathbf{x}}{d t}=A \mathbf{x}, \mathbf{y}=C \mathbf{x}
$$

avec $\mathbf{x} \in \mathbb{R}^{n}, \mathbf{y} \in \mathbb{R}^{p}, A \in \mathbb{R}^{n} \times \mathbb{R}^{n}$ et $C \in \mathbb{R}^{p} \times \mathbb{R}^{n}$. En considérant l'état $\mathbf{x}$ du systéme défini dans $\mathcal{X}=\mathbb{R}^{n}$ et la sortie y définie dans $\mathcal{Y}=\mathbb{R}^{p}$, les transformations locales de groupe suivantes permettent de montrer que la modélisation est G-invariante et G-équivariante. Ainsi, $\forall g=\mathbf{x}_{0} \in G$ :

$$
\varphi_{g}(\mathbf{x})=\mathbf{x}_{0} \cdot \mathbf{x}, \quad \psi_{g}(\mathbf{u})=0, \quad \rho_{g}(\mathbf{y})=\mathbf{x}_{0} \cdot \mathbf{y}
$$

Nous considérons ici la propriété de linéarité de l'action du groupe de transformations.

Definition 1.5. Equation de normalisation : Pour deux points quelconques $x, c \in \mathcal{T}$, il existe g tel que $\theta_{g}(x)=L_{g}(x)=g x=$ cou $\theta_{g}(x)=R_{g^{-1}}(x)=x g^{-1}=c$. Ainsi l'existence d'un repère mobile noté par la suite $\gamma$ en tout point $x \in \mathcal{T}$ est garantie. En particulier, en choisissant $c=e$, nous en déduisons que $\gamma(x)=x^{-1}$. Cette procédure est appelée "normalisation».

Definition 1.6. Un pre-filtre G-invariant pour le système $G$-invariant $\frac{d \mathbf{x}}{d t}=f(\mathbf{x}, \mathbf{u})$ avec une sortie G-équivalente $\mathbf{y}=h(\mathbf{x}, \mathbf{u})$ s'écrit :

$$
\dot{\hat{\mathbf{x}}}=f(\hat{\mathbf{x}}, \mathbf{u})+\sum_{i=1}^{n} \mathbf{K}_{i}(I(\hat{\mathbf{x}}, \mathbf{u}), E) \cdot w_{i}(\hat{\mathbf{x}})
$$

où le gain matriciel $\mathbf{K}_{i}$ dépend de la trajectoire du système seulement au travers de l'ensemble complet d'invariant connu $I(\hat{\mathbf{x}}, \mathbf{u})=\psi_{\hat{\mathbf{x}}^{-1}}(\mathbf{u}) \cdot w_{i}(\hat{\mathbf{x}}):=\left[D \varphi_{\gamma(\hat{\mathbf{x}})}(\hat{\mathbf{x}})\right]^{-1} \cdot \partial / \partial \mathbf{x}_{i}$ est un vecteur invariant qui projette l'ensemble des termes de correction invariants sur chaque composante de l'équation d'évolution $f(\hat{\mathbf{x}}, \mathbf{u})$, c'est-à-dire l'espace tangent. $\partial / \partial \mathbf{x}_{i}$ est le ième champ de vecteur de la base canonique de $\mathbb{R}^{n}$.

Les propriétés de convergence de l'équation [2] dépendent des gains $\mathbf{K}_{i}$ et de la façon dont l'erreur d'estimation sur l'état est définie. En effet, au lieu de considérer une erreur d'estimation d'état linéaire classiquement notée $\hat{\mathbf{x}}-\mathbf{x}$, la théorie des observateurs invariants définit une erreur d'estimation invariante sur l'état du système notée $\eta(\mathbf{x}, \hat{\mathbf{x}})=\mathbf{x}^{-1} \hat{\mathbf{x}}$.

Definition 1.7. La convergence asymptotique de $\hat{\mathrm{x}}$ vers $\mathrm{x}$ est équivalente à la stabilité de la dynamique de l'erreur d'état invariante qui est régie par l'équation très générale suivante :

$$
\dot{\eta}=\Upsilon(\eta, I(\hat{\mathbf{x}}, \mathbf{u}))
$$

où $\Upsilon$ est une fonction lisse (de classe $\mathcal{C}^{\infty}$ ). Ainsi, il apparaît que $\eta$ dépend de la trajectoire du système seulement au travers des invariants connus $I(\hat{\mathbf{x}}, \mathbf{u})$. 
Cette méthode de construction est systématique pour le design des (pré)-observateurs appelés aussi observateurs candidats dont les termes de correction préservent les symétries du système originel. Néanmoins, les hypothèses nécessaires qui ont été formulées quant à la régularité et aux propriétés de l'action de groupe sur l'état, c'est à $\operatorname{dire}: \operatorname{dim}\left(\varphi_{g}\right)=\operatorname{dim}(G)$, restent un verrou à lever afin que l'approche devienne complètement systématique car dans ce cas là nous ne pouvons pas appliquer le théorème des fonctions implicitent et résoudre les équations de normalisation.

Revenons sur l'exemple du système linéaire invariant. Nous considérons donc que les équations de ce système sont $\mathrm{G}$-invariante et $\mathrm{G}$-équivariante. Le groupe de transformation définit auparavant est tel que $\operatorname{dim}(G)=\operatorname{dim}(\mathcal{X})=n$, ce qui permet d'identifier et d'assimiler $G$ et $\mathcal{X}$. Identifiant $G$ à $\mathcal{R}^{*}$ à partir du cas particulier où $g=\mathrm{x}^{-1}$, nous obtenons, via les équations de normalisation :

$$
\varphi_{\mathbf{x}^{-1}}(\mathbf{x})=\mathbf{x} \cdot \mathbf{x}^{-1}=0=e
$$

Nous considérons une erreur de sortie construite autour de la transformation invariante de sortie $\rho_{g}(\mathbf{y})$ et de la solution de l'équation de normalisation notée $\gamma$ avec $\gamma(\mathbf{x})=\mathbf{x}^{-1}$ telle que :

$$
\mathbf{E}=\rho_{\gamma_{\left(\mathbf{x}_{0}\right)}}(\hat{\mathbf{y}})-\rho_{\left.\gamma_{\left(\mathbf{x}_{0}\right)}\right)}(\mathbf{y})=\mathbf{x}^{-1} \hat{\mathbf{y}}-\mathbf{x}^{-1} \mathbf{y}=\mathbf{x}^{-1}(\hat{\mathbf{y}}-\mathbf{y})
$$

On construit un observateur invariant pour le système linéaire que nous considérons en utilisant les $n$ champs de vecteurs invariants suivants :

$$
w_{i}(\hat{\mathbf{x}}):=\left[D \varphi_{\gamma(\hat{\mathbf{x}})}(\hat{\mathbf{x}})\right]^{-1} \cdot \partial / \partial \mathbf{x}_{i}=\mathbf{x} \cdot \partial / \partial \mathbf{x}_{i}, \quad \forall i \in\{1, \cdots, n\}
$$

On obtient alors l'observateur :

$$
\begin{aligned}
& \dot{\hat{\mathbf{x}}}=A \hat{\mathbf{x}}+\sum_{i=1}^{n} \mathbf{K}_{i} \mathbf{E} \cdot w_{i}(\hat{\mathbf{x}})=A \hat{\mathbf{x}}+\mathbf{K}(\mathbf{y}-\hat{\mathbf{y}}) \\
& \hat{\mathbf{y}}=C \hat{\mathbf{x}}
\end{aligned}
$$

avec $\mathbf{K} \in \mathbb{R}^{n} \times \mathbb{R}^{p}$ une matrice de gain. $\operatorname{Si}(A, C)$ est observable ( au sens du critère de rang de Kalman), alors on peut choisir $\mathrm{K}$ de manière à obtenir un observateur convergent. Cet exemple nous permet de retrouver l'écriture classique d'un observateur pour un système linéaire stationnaire.

\subsection{Quelques remarques sur l'erreur d'estimation}

Les propriétés d'invariance d'un estimateur d'état, que l'on souhaite voir préserver les symétries intrinsèques au système, reposent essentiellement sur la notion d'erreur d'estimation sur l'état invariante et sur sa dynamique propre. En effet, il est possible de montrer que cette dernière dépend uniquement de l'erreur d'estimation sur l'état invariante elle-même et de l'ensemble complet d'invariants fondamentaux du système défini par $I(\hat{\mathbf{x}}, \mathbf{u})=\psi_{\gamma(\hat{\mathbf{x}})}(\mathbf{u})=\psi_{\hat{\mathbf{x}}^{-1}}(\mathbf{u})$. Cette dynamique dépend des trajectoires estimées par le système uniquement au travers de la non-linéarité $I$.

Il s'agit là d'une grande différence par rapport à la plupart des algorithmes d'estimation non linéaires conventionnels tels que l'EKF ou l'UKF pour lesquels la dynamique de l'erreur d'estimation dépend directement de la trajectoire suivie par l'engin. Ceci étend donc le domaine de convergence des observateurs invariants et en fait des estimateurs plus robustes. Ainsi, pour un système dynamique qui suit une 
trajectoire de référence quasi-permanente, i.e. tels que les invariants fondamentaux $I(\hat{\mathbf{x}}, \mathbf{u})$ ne dépendent pas du temps, $I(\hat{\mathbf{x}}, \mathbf{u})=c$, il peut être démontré que les matrices de gain $\overline{\mathbf{K}}$ convergent vers des valeurs fixes pour toutes trajectoires $\mathbf{x}^{\prime}$ telles que $I\left(\mathbf{x}^{\prime}, \mathbf{u}\right)=c$. Par la suite, il peut être démontré que, pour certain système comme le système de l'AHRS (Attitude and Heading Reference System), et sous certaines conditions de découplage de l'estimation des composantes du vecteur d'état $\mathrm{x}$, la dynamique de l'erreur d'estimation sur l'état invariante $\eta(\mathbf{x}, \hat{\mathbf{x}})$ obéit à une équation dite autonome c'est-à-dire de la forme : $\dot{\eta}=\Upsilon(\eta, c)$. Un tel filtre sera caractérisé par un large domaine de convergence pour différents réglages des valeurs des gains.

Pour pallier à la difficulté de régler les gains de correction manuellement, l'état de l'art du domaine rapporte qu'un algorithme de type EKF, généralisé, a été développé pour obtenir ces derniers. Celui-ci repose sur une linéarisation de la dynamique de l'erreur d'estimation sur l'état invariante qui permet d'identifier des matrices d'évolution $(\mathbf{A}(\hat{I}))$ et d'observation $(\mathbf{C}(\hat{I}))$ dépendantes des invariants du problème d'estimation. Par la suite, les équations du filtrage de Kalman étendu peuvent être appliquées au système des erreurs invariantes linéarisées de sorte que la résolution de l'équation de Riccati soit menée avec le couple $(\mathbf{A}(\hat{I}), \mathbf{C}(\hat{I}))$. La démarche suivie dans l'IEKF revient à formuler mathématiquement un EKF classique mais pour une erreur d'estimation sur l'état invariante. L'IEKF fournit une approximation, au $2^{\text {nd }}$ ordre, de l'erreur d'estimation sur l'état invariante. Malgré ses nombreux avantages, cette technique peut être relativement complexe à exploiter du fait de la linéarisation qu'elle nécessite pour identifier les matrices $\mathbf{A}(\hat{I})$ et $\mathbf{C}(\hat{I})$. Afin de s'affranchir de ce point difficile, des recherches ont conduit au développement d'une méthode différente pour l'estimation de l'état des systèmes dynamiques dans un cadre non linéaire. Tout comme pour l'IEKF, l'IUKF (Invariant Unscented Kalman Filter) a cherché à conférer à l'estimateur les mêmes propriétés d'invariance que celles qui peuvent caractériser le système observé. Ces deux méthodes sont présentées dans les sections suivantes.

\subsection{Le filtrage de Kalman Etendu invariant : I'IEKF}

La démarche mise en place par $\mathrm{S}$. Bonnabel et P. Rouchon dans le développement de l'IEKF (en anglais Invariant Extented Kalman Filter) consiste à retrouver une formulation de l'EKF classique en intégrant la théorie des observateurs invariants, c'est-à-dire retrouver les matrices linéarisées de la dynamique de l'erreur d'estimation sur l'état invariante associée au système considéré. La notion de covariance qui constitue l'inconnue de l'équation de Riccati à résoudre se trouve redéfinie et correspond désormais à une matrice de covariance pour les erreurs invariantes. Pour mémoire, nous rappelons ci-après les équations de l'EKF «stantard» dans le cas d'une dynamique continue :

$$
\begin{gathered}
\dot{\hat{\mathbf{x}}}=f(\hat{\mathbf{x}}, \mathbf{u})+\mathbf{K}(t)\left(\mathbf{y}_{m}-h(\hat{\mathbf{x}}, \mathbf{u})\right) \\
\mathbf{K}(t)=\mathbf{P}(t) \mathbf{C}^{T}(t)\left(\mathbf{N N}^{T}\right)^{-1} \\
\dot{\mathbf{P}}(t)=\mathbf{A}(t) \mathbf{P}(t)+\mathbf{P}(t) \mathbf{A}^{T}(t)-\mathbf{P}(t) \mathbf{C}^{T}(t)\left(\mathbf{N N}^{T}\right)^{-1} \mathbf{C}(t) \mathbf{P}(t)+\mathbf{M M}^{T}
\end{gathered}
$$

Le calcul des gains de correction pour l'estimateur invariant à partir des principes de l'EKF débute tout d'abord par une linéarisation, au second ordre, de l'erreur d'estimation sur l'état invariante, $\eta(\mathbf{x}, \hat{\mathbf{x}})=$ $\varphi_{\mathbf{x}^{-1}}(\hat{\mathbf{x}})-\varphi_{\mathbf{x}^{-1}}(\mathbf{x})$, pour identifier les matrices $\mathbf{A}, \mathbf{C}, \mathbf{N}$ et $\mathbf{M}$. La donnée de celle-ci permet ensuite d'obtenir la matrice des gains $\mathbf{K}$, par les équations [4] à [6], et donc de connaître la dynamique de l'erreur linéarisée $\Delta \ddot{\eta}=(\mathbf{A}-\mathbf{K C}) \Delta \dot{\eta}$. Cela permet donc in fine d'écrire les équations de l'IEKF de la manière suivante : 


$$
\begin{aligned}
& \dot{\hat{\mathbf{x}}}=f(\hat{\mathbf{x}}, \mathbf{u})+\overbrace{D L_{\hat{\mathbf{x}}}} \mathbf{K}(t) \cdot \overbrace{\left(\rho_{\hat{\mathbf{x}}^{-1}}(\mathbf{z})-\rho_{\hat{\mathbf{x}}^{-1}}(h(\hat{\mathbf{x}}, \mathbf{u}))\right)}^{\substack{\text { repère invariant } \\
\text { au point } \hat{\mathbf{x}} \sim w(\hat{\mathbf{x}})}} \\
& \mathbf{K}(t)=\mathbf{P}(t) \mathbf{C}^{T}(t)\left(\mathbf{N N}^{T}\right)^{-1} \\
& \dot{\mathbf{P}}(t)=\mathbf{A}(t) \mathbf{P}(t)+\mathbf{P}(t) \mathbf{A}^{T}(t)-\mathbf{P}(t) \mathbf{C}^{T}(t)\left(\mathbf{N N}^{T}\right)^{-1} \mathbf{C}(t) \mathbf{P}(t)+\mathbf{M M}^{T}
\end{aligned}
$$

Dans l'équation [7], $D L_{\hat{\mathbf{x}}}$ signifie que l'invariance est considérée à gauche sur le groupe de Lie $G$ mais nous pourrions tout aussi bien considérer une invariance à droite $D R_{\hat{\mathbf{x}}}$. A noter que l'élaboration d'observateurs invariants sur un groupe de Lie fait ici l'hypothèse que l'action du groupe est libre ${ }^{1}$ sur l'espace d'état, et que celle-ci a la même dimension que $\mathcal{X}$. Il n'y a donc qu'une seule orbite et l'action de groupe est dite transitive : les observateurs invariants développés pour les problématiques AHRS et INS obéissent à cette hypothèse.

Considérons connue une erreur d'estimation sur l'état invariante $\eta$ vérifiant : $\dot{\eta}=D g_{u t}(\eta)$ où $D g_{u t}$ est un champ de vecteur. Puisque $G$ constitue une variété différentiable, il est possible d'identifier un morceau de l'algèbre de Lie de $G$ à un voisinage $U$ centré sur l'élément neutre $e$ du groupe grâce à l'application exponentielle. Ainsi, une erreur $\eta$ petite, proche de $e$, peut être assimilée à un élément de l'algèbre de Lie, noté $\xi$, dans le sens où il est possible d'écrire $\eta_{t}=\exp \left(\epsilon \xi_{t}\right)$ où $\epsilon \in \mathbb{R}$ est tel que $\epsilon \lll 1$. Jusqu'aux termes d'ordre 2 en $\epsilon$, nous avons donc l'équation de l'erreur d'estimation sur l'état invariante $\eta_{t}$ linéarisée dans l'espace tangent à $e$ qui s'écrit :

$$
\frac{d}{d t} \xi=[\xi, f(e, \hat{I})]-\frac{\partial f}{\partial u}(e, \hat{I}) \frac{\partial \psi}{\partial g}(e, \hat{I}) \xi-\sum_{i=1}^{n}\left(\frac{\partial \mathbf{K}_{i}}{\partial \mathrm{E}}(\hat{I}, 0) \frac{\partial h}{\partial \mathbf{x}}(e, \hat{I}) \xi\right) w_{i}
$$

où $[$, ] désigne le crochet de Lie. Les matrices $\mathbf{A}(t)$ et $\mathbf{C}(t)$ peuvent être identifiées grâce à l'équation [10] de la manière suivante :

$$
\begin{gathered}
\mathbf{A}(\hat{I}): \xi \mapsto[\xi, f(e, \hat{I})]-\frac{\partial f}{\partial u}(e, \hat{I}) \frac{\partial \psi}{\partial h}(e, \hat{I}) \xi \\
\mathbf{C}(\hat{I})=\frac{\partial h}{\partial x}(e, \hat{I}), \quad M=M(e), \quad N=N(e) .
\end{gathered}
$$

Il a été montré dans (Barrau et al. [2015]) que l'approximation en série de Taylor au $1^{\mathrm{er}}$ ordre de l'équation [10], ré-écrite sous la forme $\dot{\xi}=D g_{u t}(\xi, e)$, est représentative du comportement global de la dynamique de l'erreur d'estimation sur l'état invariante. Ce résultat a amené l'énoncé du théorème qui suit :

Theorem 1.8. Considérons l'erreur d'estimation sur l'état invariante $\eta_{t}^{*}$, définie pour une dynamique invariante à droite ou à gauche, l'exposant $(*)$ désignant indifféremment $L$ ou $R$. Soit $\xi_{0}^{*} \in \mathbb{R}^{\operatorname{dim}(g)}$ tel que $\eta_{0}^{*}=\exp \left(\xi_{0}\right)$. Si $\xi_{t}^{*}$ est défini pour $t>0$ par une équation différentielle linéaire de la forme :

$$
\frac{d}{d t} \xi_{t}^{*}=A_{t}^{*} \xi_{t}^{*} \text { où }: A_{t}^{*}:=D g_{u t}^{*}(e)
$$

alors nous avons :

$$
\forall t \geq 0, \eta_{t}^{*}=\exp \left(\xi_{t}^{*}\right)
$$


Le théorème 1 est démontré intégralement dans Barrau et al. [2014]. Il est extrêmement important car il garantit que l'étape de prédiction effectuée par n'importe quel filtre de Kalman exploitant une erreur d'état invariante ${ }^{2}$, et donc implicitement une carte exponentielle de l'espace géométrique, sera menée de façon à respecter l'évolution vraie en terme de covariance de l'erreur sur l'état estimé.

\subsection{Le filtrage de Kalman Uscented invariant : I'IUKF}

Dans cette section, nous proposons une autre modification de l'UKF classique, pour des systèmes dynamiques possédant des symétries. Comme nous l'avons mentionné en introduction, l'UKF présente l'avantage de calculer une solution approchée au problème d'estimation non linéaire optimal de l'état d'un système dynamique, en temps discret, sans recourir à une quelconque linéarisation d'équations différentielles. La technique dénommée « approche par sigma point» (en anglais, Sigma Point Approach - SPA) est habituellement utilisée pour déterminer l'approximation du processus aléatoire gaussien $x_{k \mid k}$, au cours du temps, sur lequel sont appliquées des transformations non linéaires tel que :

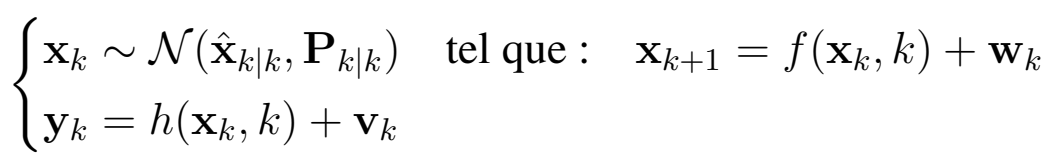

où $\mathbf{x} \in \mathbb{R}^{n}, \mathbf{y} \in \mathbb{R}^{p}$ et $\mathbf{w}, \mathbf{v}$ sont des bruits blancs gaussiens. Rappelons que le but de la technique SPA est de définir, au cours du temps, un nombre fini de sigma points $\mathcal{X}_{k \mid k}$ qui capturent la vraie moyenne $\hat{\mathbf{x}}_{k \mid k}$ et la vraie covariance $\mathbf{P}_{k \mid k}$ de la distribution de probabilité caractérisant $\mathbf{x}_{k \mid k}$. Dans le cas gaussien, cet ensemble de points doit représenter précisément les deux premiers moments de la variable aléatoire. Ces sigma points sont ensuite propagés à travers les fonctions non linéaires $f$ et $h$, apparaissant dans l'équation [13]. Cette propagation fournit un nuage de points modifiés par rapport à la répartition initiale des sigma points. La moyenne $\hat{\mathbf{x}}_{k+1 \mid k}$ et la covariance $P_{k+1 \mid k}$ estimées associées aux points transformés sont ensuite calculées par rapport à la statistique de ce nouvel ensemble de points. L'UKF peut être considéré comme une simple extension de la technique SPA qui permet donc de calculer les approximations du problème d'estimation dans le cas où :

$$
\begin{aligned}
\mathbf{x}_{k+1} & =f\left(\mathbf{x}_{k}, \mathbf{u}_{k}\right)+\mathbf{w}_{k} \\
\mathbf{y}_{k} & =h\left(\mathbf{x}_{k}, \mathbf{u}_{k}\right)+\mathbf{v}_{k}
\end{aligned}
$$

Comme cela est présenté dans la référence (Barrau et al. [2015]), nous considérerons que les bruits d'évolution et d'observation ( $\mathbf{w}$ et $\mathbf{v}$ ) entrent dans le système de façon invariante. Ces bruits peuvent être considérés comme des entrées exogènes qui ne remettent pas en cause l'existence même de symétries propres au système. Afin donc de préserver celles-ci, leurs insertions respectives dans les équations d'évolution et d'observation du système se fait au travers de termes $G$-invariant/équivariant.

Lorsque la dynamique du système observé présente des propriétés d'invariance par symétries, les équations de base du SRUKF ne permettent pas de synthétiser un estimateur de l'état du système disposant de propriétés analogues. Pourtant, du point de vue de la convergence, il peut être très intéressant de chercher à conférer à tout filtre estimateur candidat les propriétés d'invariance du système, à l'instar de ce qui est fait pour les observateurs invariants et l'IEKF. Ainsi, JP. Condomines, C. Seren et G. Hattenberger ont modifié l'algorithme SRUKF afin que cela soit le cas également. A partir des principes mis en œuvre et

2.On peut écrire $\eta=x^{-1} \hat{x}$ pour une dynamique invariance à gauche ou $\eta=\hat{x} x^{-1}$ pour une dynamique invariance à droite. 
des multiples étapes de calcul qui fondent l'algorithme du SRUKF, une réécriture naturelle des équations, visant à adapter la méthode à un cadre invariant pour l'estimation, peut être obtenue simplement en redéfinissant les termes d'erreur utilisés dans la version standard présentée au cours de la section précédente. En effet, les termes d'erreur linéaires $\hat{\mathbf{x}}-\mathbf{x}$ et $\mathbf{z}-\hat{\mathbf{y}}$, classiquement rencontrés en filtrage de Kalman, ne préservent en rien les symétries et les invariances du système. Ainsi, nous considérerons les erreurs d'état et de sortie invariantes suivantes :

$$
\left\{\begin{array}{l}
\hat{\mathbf{x}}-\mathbf{x} \longrightarrow \eta(\mathbf{x}, \hat{\mathbf{x}})=\varphi_{\gamma(\mathbf{x})}(\mathbf{x})-\varphi_{\gamma(\mathbf{x})}(\hat{\mathbf{x}}) \\
\mathbf{z}-\hat{\mathbf{y}} \longrightarrow \mathrm{E}(\mathbf{z}, \hat{\mathbf{x}}, \hat{\mathbf{y}})=\rho_{\gamma(\hat{\mathbf{x}})}(\hat{\mathbf{y}})-\rho_{\gamma(\hat{\mathbf{x}})}(\mathbf{z})
\end{array}\right.
$$

Dans l'équation [16], $\mathrm{x}$ désigne l'état « réel » du système. $\hat{\mathrm{x}}$ correspond à l'estimé de cet état. Les notations $\hat{\mathbf{y}}$ et $\mathbf{z}$ indiquent respectivement la sortie estimée et le vecteur des mesures bruitées.

Remarque : on peut vérifier aisément que ces erreurs sont invariantes. En effet, $\forall g \in G$ on a :

$$
\begin{aligned}
\eta\left(\varphi_{g}(\mathbf{x}), \varphi_{g}(\hat{\mathbf{x}})\right) & =\varphi_{\gamma\left(\varphi_{g}(\mathbf{x})\right)}\left(\varphi_{g}(\mathbf{x})\right)-\varphi_{\gamma\left(\varphi_{g}(\mathbf{x})\right)}\left(\varphi_{g}(\hat{\mathbf{x}})\right) \\
& =\varphi_{\gamma\left(\varphi_{g}(\mathbf{x})\right) g}(\mathbf{x})-\varphi_{\gamma\left(\varphi_{g}(\mathbf{x})\right) g}(\hat{\mathbf{x}}) \text { par définition de la loi de composition sur } G \\
& =\varphi_{\gamma(\mathbf{x})}(\mathbf{x})-\varphi_{\gamma(\mathbf{x})}(\hat{\mathbf{x}}) \operatorname{car} \gamma\left(\varphi_{g}(\mathbf{x})\right) g=\gamma(\mathbf{x}) \\
& =\eta(\mathbf{x}, \hat{\mathbf{x}})
\end{aligned}
$$

et :

$$
\begin{aligned}
\mathrm{E}\left(\rho_{g}(\mathbf{z}), \varphi_{g}(\hat{\mathbf{x}}), \rho_{g}(\hat{\mathbf{y}})\right) & =\rho_{\gamma\left(\varphi_{g}(\hat{\mathbf{x}})\right)}\left(\rho_{g}(\hat{\mathbf{y}})\right)-\rho_{\gamma\left(\varphi_{g}(\hat{\mathbf{x}})\right)}\left(\rho_{g}(\mathbf{z})\right)=\rho_{\gamma\left(\varphi_{g}(\hat{\mathbf{x}})\right) g}(\hat{\mathbf{y}})-\rho_{\gamma\left(\varphi_{g}(\hat{\mathbf{x}})\right) g}(\mathbf{z}) \\
& =\rho_{\gamma(\hat{\mathbf{x}})}(\hat{\mathbf{y}})-\varphi_{\gamma(\hat{\mathbf{x}})}(\mathbf{z}) \\
& =\mathbf{E}(\mathbf{z}, \hat{\mathbf{x}}, \hat{\mathbf{y}})
\end{aligned}
$$

Dans le cas où l'action de groupe est de rang plein et transitive (i.e. telle que $\operatorname{dim}(G)=\operatorname{dim}(\mathcal{X})=n)$, nous avons vu que $G$ peut être identifié à l'espace d'état $\mathcal{X}=\mathbb{R}^{n}$ de sorte que la transformation locale $\varphi_{g}$ puisse être assimilée à une multiplication à gauche telle que $\varphi_{g}(\mathbf{x})=g \cdot \mathbf{x}$. La résolution des équations de normalisation telle que $\varphi_{g}(\mathbf{x})=g \cdot \mathbf{x}=e$, où $e$ désigne l'élément neutre du groupe $G$, conduit au repère mobile solution suivant : $\gamma(\mathbf{x})=\mathbf{x}^{-1}$. Dans ce cas, les erreurs de l'équation [16] deviennent :

$$
\left\{\begin{array}{l}
\eta(\mathbf{x}, \hat{\mathbf{x}})=\varphi_{\mathbf{x}^{-1}}(\mathbf{x})-\varphi_{\mathbf{x}^{-1}}(\hat{\mathbf{x}})=e-\mathbf{x}^{-1} \cdot \hat{\mathbf{x}} \\
\mathbf{E}(\mathbf{z}, \hat{\mathbf{x}}, \hat{\mathbf{y}})=\rho_{\hat{\mathbf{x}}^{-1}}(\hat{\mathbf{y}})-\rho_{\hat{\mathbf{x}}^{-1}}(\mathbf{z})
\end{array}\right.
$$

Dès lors, la notion de matrice de covariance des erreurs d'estimation utilisée jusqu'à maintenant en continu, dont le calcul dans l'algorithme du SRUKF peut être approché à partir de la donnée des $(2 n+1)$ sigma points $\mathcal{X}_{k \mid k}^{(i)}, i \in \llbracket 1 ;(2 n+1) \rrbracket$, construits à chaque pas de temps $k \in \mathbb{Z}^{*}$, se trouve désormais modifiée pour être associée aux erreurs définies par l'équation [18]. Nous parlerons alors de matrice de covariance des erreurs d'estimation invariantes. L'étape de décomposition de la matrice de covariance associée à l'erreur d'estimation sur l'état fait donc apparaître un ensemble de $(2 n+1)$ erreurs invariantes calculées entre chaque sigma point et l'état prédit. La notion de covariance porte donc désormais sur l'erreur d'état invariante et non plus sur un terme d'erreur linéaire. Il s'agit de la principale nuance introduite avec le filtrage de Kalman unscented classique. Hormis cette modification majeure, l'étape de prédiction n'est que peu modifiée par rapport à sa version originale. Pour ce qui est de l'étape de correction, nous voyons que les modifications apportées à la version standard du SRUKF sont plus nombreuses. En effet, la redéfinition de la notion de matrice de covariance, associant celle-ci à des erreurs d'estimation désormais invariantes, modifie : 
(1) le calcul de la covariance prédite relative à la sortie y tel que :

$$
\mathbf{P}_{\mathbf{y}, k+1 \mid k} \propto \mathbf{E}\left(\hat{\mathbf{y}}_{k+1 \mid k}, \boldsymbol{\mathcal { X }}_{k+1 \mid k}, \hat{\mathbf{Y}}_{k+1 \mid k}\right)
$$

2 et le calcul de la covariance croisée prédite entre les erreurs d'estimation portant sur l'état $\mathrm{x}$ et la sortie y tel que :

$$
\mathbf{P}_{\mathbf{x y}, k+1 \mid k} \propto \eta\left(\mathcal{X}_{k+1 \mid k}, \hat{\mathbf{x}}_{k+1 \mid k}\right), \mathrm{E}\left(\hat{\mathbf{y}}_{k+1 \mid k}, \boldsymbol{\mathcal { X }}_{k+1 \mid k}, \hat{\mathbf{Y}}_{k+1 \mid k}\right)
$$

Il s'ensuit naturellement que le gain de correction calculé pour l'estimation de l'état par une technique de type UKF dépendra, par transitivité, d'invariants fondamentaux pour le système considéré $\left(\psi_{\mathbf{x}^{-1}}(u)\right)$ et d'erreurs de sortie invariantes $(\mathrm{E}(\hat{\mathbf{y}}, \mathbf{x}, \cdot))$.

Le cadre invariant défini pour le système considéré entraine également une réécriture des équations de correction de l'état prédit. En effet, il apparaît que le terme de correction additif repose dorénavant sur : un gain qui dépend des invariants du problème d'estimation ; une innovation invariante. De plus, ce terme correctif se retrouve projeté dans le repère invariant, de sorte que, la correction de l'état prédit soit réalisée, composante par composante, i.e. selon les directions des $n$ vecteurs de la base canonique de $\mathbb{R}^{n}$ formée par le champ de vecteurs invariants $\mathcal{B}\left(\hat{\mathbf{x}}_{k+1 \mid k}\right)=\left\{\omega_{i}\left(\hat{\mathbf{x}}_{k+1 \mid k}\right)\right\}_{i \in \llbracket 1 ; n \rrbracket}$. Ce travail d'adaptation de la version standard du SRUKF constitue l'approche la plus naturelle pour dériver un estimateur invariant dont les termes de correction :

$\square$ sont calculés selon un schéma de type UKF, adapté au cadre invariant du problème d'estimation posé, qui échantillonne l'espace d'état à partir d'une technique SUT (Scaled Uunscented Transform) classique, identique à celle communément exploitée pour le filtrage de Kalman par Sigma Points ;

$\square$ respectent les symétries propres au système du fait qu'ils sont construits à partir d'une innovation invariante et de gains dépendant des invariants fondamentaux et de l'erreur de sortie invariante.

Il est à noter au passage que l'algorithme présenté précédemment repose sur un paramétrage multiple du groupe de transformations en définissant successivement chaque inverse de sigma point comme paramètre à considérer pour l'application composite $\phi_{g}=\left(\varphi_{g}, \psi_{g}, \rho_{g}\right)$. Ceci revient à définir un ensemble de $(2 n+1)$ repères mobiles, de dimension $n$, dans l'espace d'état qui envoient chaque sigma point vers l'élément neutre $e$ par l'application locale $\varphi_{g}$ qui, pour rappel, s'apparente à une action transitive de rang plein telle que $\varphi_{g}(\mathbf{x})=g \cdot \mathbf{x}$ (cf. Figure 1). L'IUKF est générique en ce sens qu'il ne se préoccupe ni de la forme des équations du modèle d'observation, ni de celle associée aux relations définissant la transformation de groupe $\rho_{g}$.

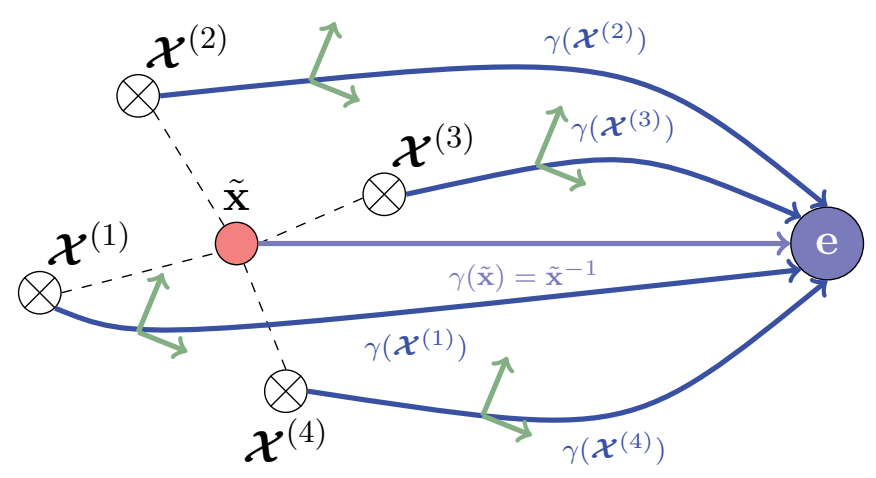

Figure 1. Réseau des $(2 n+1)$ repères invariants associés aux sigma points 


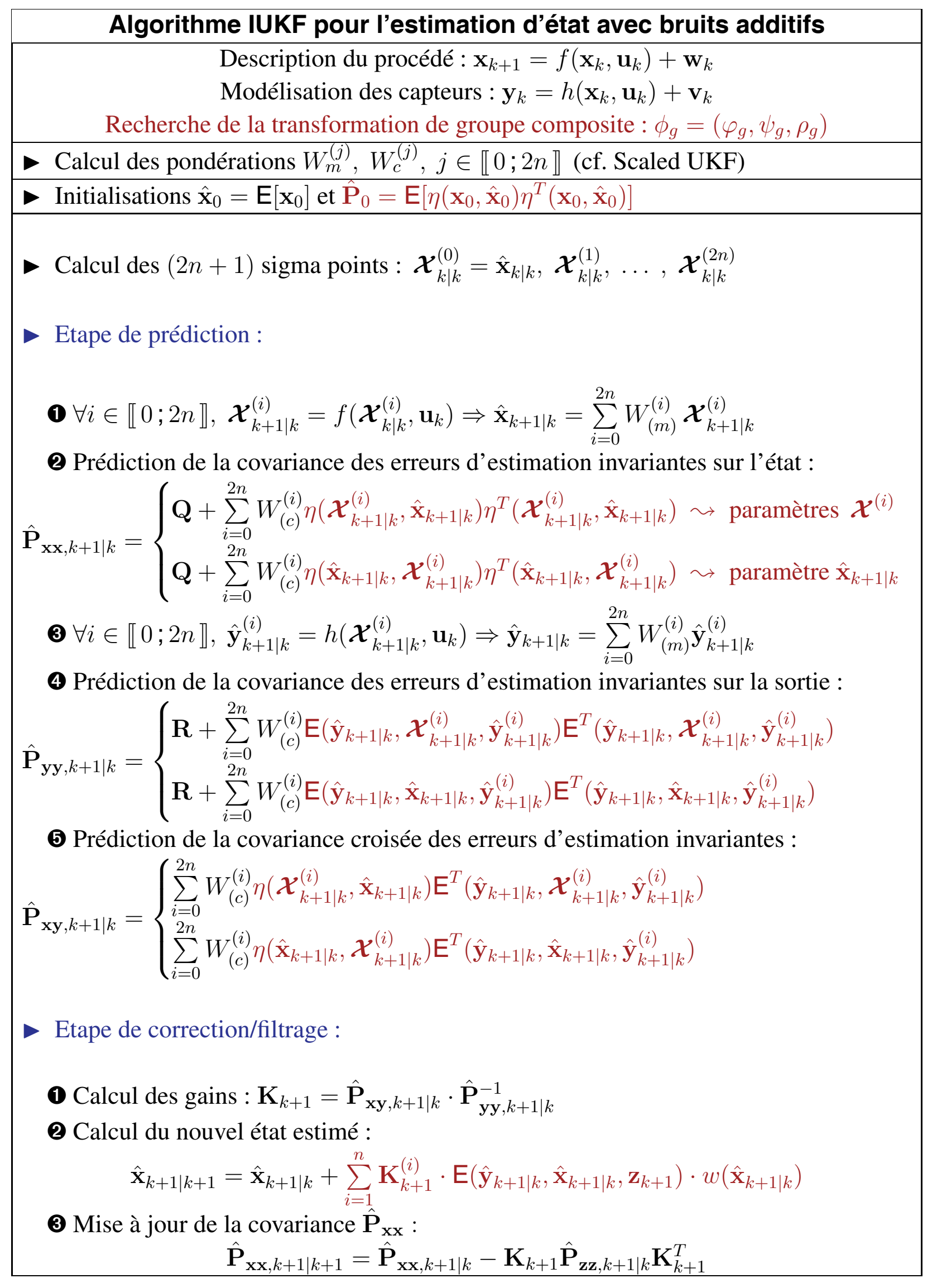




\section{Exemple de motivation pour illustrer I'IUKF et de I'IEKF}

Dans le prolongement des développements méthodologiques de l'IEKF et de l'IUKF présenté à la section précédente, nous allons nous intéresser ici aux performances de ces deux algorithmes sur un système appelé inclinomètre ou en anglais tilt sensor system. Ce système, comme son nom l'indique, permet de mesurer l'inclinaison de tout objet disposant d'un capteur mesurant l'accélération de celui-ci. D'autres système, plus complexe, comme un AHRS (Attitude and Heading Reference System), serait en mesure de réaliser la même opération mais ne nous permettrait pas de mettre en exergue les différentes propriétés intrinsèques de l'IUKF. Par ailleurs, le modèle d'observation de l'inclinomètre mérite que l'on s'y attache puisqu'il est communément utilisé dans de nombreux domaines : communication, radars, optométrie, etc. Enfin, cet exemple nous permettra d'étudier la non linéarité du modèle uniquement sur l'équation d'observation. Comme nous le verrons par la suite, l'erreur de sortie usuelle (i.e. li-

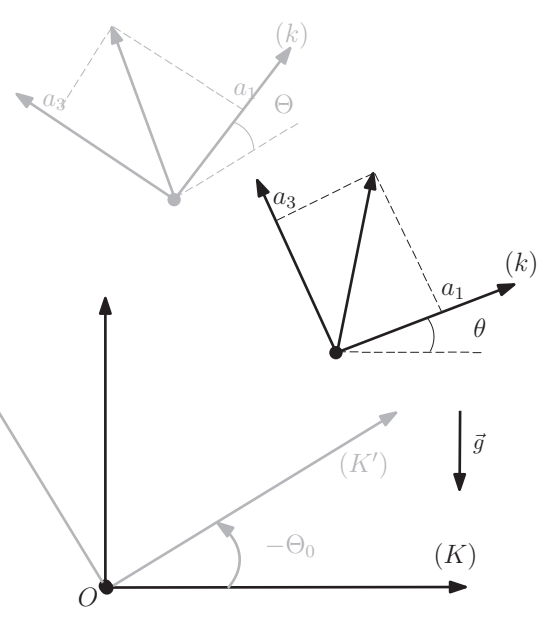

Figure 2. La dynamique de l'inclinomètre est invariante sous l'action du groupe $S O(2)$ néaire), calculée à partir de cette équation, ne préserve pas la géométrie du système. Le concept d'erreur de sortie invariante est une idée clé dans la construction de tout filtre invariant. Nous mettrons donc en avant cette propriété en comparant l'UKF et l'IUKF. Ainsi, pour estimer l'angle d'attitude d'un objet, il nous sera nécessaire de disposer de trois accéléromètres donnant une mesure de l'accélération spécifique de l'objet notée $a_{m}=\left(a_{1}, a_{2}, a_{3}\right)$. Nous considérons ici plus particulièrement l'estimation de l'angle $\theta$, qui décrit la rotation de l'objet dans le plan vertical comme le montre la Figure 2. Le modèle d'observation non linéaire de l'inclinomètre, calculé à l'aide de la matrice de passage DCM, s'écrit de la manière suivante :

$$
\left(\begin{array}{l}
y_{a_{1}} \\
y_{a_{3}}
\end{array}\right)=\left(\begin{array}{c}
-\sin (\theta) \\
\cos (\theta)
\end{array}\right)=h(\mathbf{x})
$$

A ce stade, il est nécessaire de formuler une hypothèse sur l'angle d'attitude de l'engin afin d'obtenir l'équation d'évolution du système. Nous supposons que l'angle ne change pas durant la période de mesure ou autrement dit que la dynamique de l'angle est constante. L'équation d'évolution s'écrit donc :

$$
\dot{\theta}=0
$$

Nous pouvons voir que l'espace d'état $\mathcal{X}=\mathcal{S}^{1}$ coïncide topologiquement avec $\mathrm{SO}(2)$ comme cela est illustré par la Figure 2. La dynamique du système est donc indépendante de l'orientation du repère de référence choisi et donc invariante sous l'action du groupe $S O(2)$ des rotations du plan. En considérant les équations d'évolution et d'observation du modèle de l'inclinomètre, les transformations locales de groupe suivantes permettent de montrer que la modélisation est G-invariante et G-équivariante. Notons les représentations d'état non linéaire décrites sous forme compacte telle que $\dot{\mathbf{x}}=f(\mathbf{x}, \mathbf{u})$ et $\mathbf{y}=h(\mathbf{x}, \mathbf{u})$ où $\mathbf{x}=\theta$ et $\mathbf{y}=\left(y_{a_{1}}, y_{a_{3}}\right)^{T}$. Ainsi, $\forall g=\theta_{0} \in G$ :

$$
\varphi_{g}(\mathbf{x})=\left(\theta+\theta_{0}\right), \quad \psi_{g}(\mathbf{u})=0, \quad \rho_{g}(\mathbf{y})=\left(\begin{array}{l}
y_{a_{1}} \cos \theta_{0}-y_{a_{3}} \sin \theta_{0} \\
y_{a_{3}} \cos \theta_{0}+y_{a_{1}} \sin \theta_{0}
\end{array}\right)
$$


qui s'écrit avec les nouvelles variables de la manière suivante :

$$
\rho_{g}\left(y_{a_{1}}, y_{a_{3}}\right)=\left(\begin{array}{c}
y_{a_{1}} \cos \theta_{0}-y_{a_{3}} \sin \theta_{0} \\
y_{a_{3}} \cos \theta_{0}+y_{a_{1}} \sin \theta_{0}
\end{array}\right)=\left(\begin{array}{c}
-\sin \left(\theta+\theta_{0}\right) \\
\cos \left(\theta+\theta_{0}\right)
\end{array}\right)=h\left(\varphi_{g}(\mathbf{x})\right)
$$

\subsection{Construction d'une erreur de sortie invariante}

Nous considérons donc les équations [2] et [1], $G$-invariant et $G$-équivariant. Le groupe de transformation définit auparavant est tel que $\operatorname{dim}(G)=\operatorname{dim}(\mathcal{X})=n=1$ ce qui permet d'identifier et d'assimiler $G$ et $\mathcal{X}$. Les actions seront également de rang plein i.e., telles que $\operatorname{dim}\left(\operatorname{Im}\left(\varphi_{g}\right)\right)=\operatorname{dim}(G)=1$. Identifiant $G$ à $S^{1}$ à partir du cas particulier où $g=\mathrm{x}^{-1}=(-\theta)$, nous obtenons, via les équations de normalisation :

$$
\varphi_{\mathbf{x}^{-1}}(\mathbf{x})=(\theta-\theta)=0=e
$$

où $e$ est l'élément neutre du groupe $G$ muni de l'addition. Les résultats de l'équation de groupe permettent ensuite de définir l'ensemble complet de $m+p$ invariants fondamentaux à partir de la paramétrisation $g=\mathbf{x}^{-1} \in G$. En effet, $\psi_{\mathbf{x}^{-1}}(u)$ fournira $m$ invariant fondamentaux pour la dynamique du système et $\rho_{\mathbf{x}^{-1}}(y), p$ invariant fondamentaux pour l'observation de celui-ci. Ainsi pour construire une erreur de sortie invariante, nous ne considérons plus l'erreur de sortie linéaire usuelle $\hat{y}-y=h(\hat{\mathbf{x}}, u)-$ $y$ qui ne préserve pas la symétrie du système pour l'estimation, mais une erreur de sortie invariante construite autour de la transformation invariante de sortie $\rho_{\left(\theta_{0}\right)}\left(y_{a_{1}}, y_{a_{3}}\right)$ et de la solution de l'équation de normalisation notée $\gamma$ (repère mobile) telle que :

$$
\gamma(x)=-\theta
$$

Une erreur de sortie invariante s'écrit donc :

$$
\begin{aligned}
E & =\rho_{\gamma_{\left(\theta_{0}\right)}}\left(\hat{y}_{a_{1}}, \hat{y}_{a_{3}}\right)-\rho_{\gamma_{\left(\theta_{0}\right)}}\left(y_{a_{1}}, y_{a_{3}}\right) \\
& =\left(\begin{array}{cc}
\cos \theta_{0} & -\sin \theta_{0} \\
\sin \theta_{0} & \cos \theta_{0}
\end{array}\right)\left(\begin{array}{l}
\hat{y}_{a_{1}} \\
\hat{y}_{a_{3}}
\end{array}\right)-\left(\begin{array}{cc}
\cos \theta_{0} & -\sin \theta_{0} \\
\sin \theta_{0} & \cos \theta_{0}
\end{array}\right)\left(\begin{array}{l}
y_{a_{1}} \\
y_{a_{3}}
\end{array}\right)=\left(\begin{array}{cc}
\cos \hat{\theta} & \sin \hat{\theta} \\
-\sin \hat{\theta} & \cos \hat{\theta}
\end{array}\right)\left(\begin{array}{l}
\hat{y}_{a_{1}}-y_{a_{1}} \\
\hat{y}_{a_{3}}-y_{a_{3}}
\end{array}\right)
\end{aligned}
$$

Ainsi, conformément aux symétries du système, l'erreur de sortie invariante peut être interprétée comme une erreur de sortie classique projetée dans un repère de Frenet (multiplication par une matrice de rotation). A noter que ce résultat est le même que celui utilisé pour découpler les entrées/sorties d'une

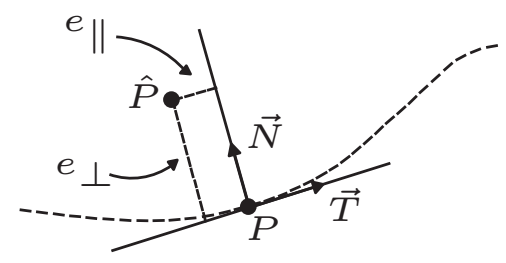

Figure 3. Interprétation des erreurs invariantes entre la position réelle $P$ et estimée $\hat{P}$

erreur de suivi de trajectoire. En effet, en considérant comme $\hat{P}$ la position à estimer et $P$ la position réelle (cf. Figure 3), nous obtenons comme sortie les deux erreurs suivantes :

$$
e_{\|}=(\hat{P}-P) \cdot \vec{T} \quad \text { et } \quad e_{\perp}=(\hat{P}-P) \cdot \vec{N}
$$


avec :

$$
\vec{T}=\left(\begin{array}{c}
\cos \theta \\
-\sin \theta
\end{array}\right) \quad \text { et } \quad \vec{N}=\left(\begin{array}{c}
\sin \theta \\
\cos \theta
\end{array}\right)
$$

Nous retrouvons bien les mêmes erreurs de sorties invariantes (cf.Martin [2004]).

Ainsi l'équation du filtre invariant s'écrira :

$$
\dot{\hat{\theta}}=0+\overline{\mathbf{K}}\left(\begin{array}{cc}
\cos \hat{\theta} & \sin \hat{\theta} \\
-\sin \hat{\theta} & \cos \hat{\theta}
\end{array}\right)\left(\begin{array}{l}
\hat{y}_{a_{1}}-y_{a_{1}} \\
\hat{y}_{a_{3}}-y_{a_{3}}
\end{array}\right)
$$

où $\overline{\mathbf{K}}$ est une matrice $1 \times 2$ de gains dépendant ici uniquement de l'erreur de sortie invariante $E$ construite précédemment.

Comme tout modèle utilisé par l'algorithme IUKF, celui-ci doit être exprimé en temps discret, soit à l'aide d'un schéma d'intégration numérique de type Runge-Kutta ou bien à partir de l'exponentielle matricielle des équations du modèle dynamique. Nous choisissons la deuxième méthode de calcul qui nous donne l'expression du modèle dynamique discret.

$$
\theta_{k}=\theta_{k-1}
$$

Afin d'obtenir un résultat le plus réaliste possible, nous supposons que les accéléromètres dont nous disposons pour la conception d'un inclinomètre ont des performances limitées et présentent donc des imperfections. Nous représentons donc les mesures fournies par les accéléromètres comme la somme d'un signal déterministe et d'un bruit blanc Gaussien de covariance $\mathbf{R}_{v}$.

Par ailleurs, un bruit d'état de covariance $Q_{\mathbf{u}}$ est introduit dans l'équation d'évolution afin d'être capable d'estimer une variation éventuelle de l'angle. En effet, si l'équation d'état n'était pas bruitée on trouverait un gain nul en régime permanent, ce qui ne permettrait plus au filtre de détecter une variation éventuelle de l'angle. Nous pouvons donc re-écrire les équations de l'inclinomètre de façon suivante :

$$
\left\{\begin{array}{l}
\theta_{k}=\theta_{k-1}+u_{k} \\
\left(\begin{array}{c}
y_{k}^{a_{1}} \\
y_{k}^{a_{3}}
\end{array}\right)=\left(\begin{array}{c}
-\sin \left(\theta_{k}\right) \\
\cos \left(\theta_{k}\right)
\end{array}\right)+\left(\begin{array}{c}
v_{k}^{a 1} \\
v_{k}^{a 3}
\end{array}\right)
\end{array}\right.
$$

Afin d'être en mesure de comparer les algorithmes entre eux, nous devons obtenir le modèle linéarisé de l'équation de mesure à partir du développement en série de Taylor de $h_{k}\left(\mathbf{x}_{k}\right)=\left(-\sin \left(\theta_{k}\right) \cos \left(\theta_{k}\right)\right)^{T}$ au voisinage de $\hat{\mathbf{x}}_{k \mid k-1}$. Ainsi, le calcul de la linéarisation donne :

$$
H_{n}=\left(\frac{\partial}{\partial \mathbf{x}} h_{k}^{T}(\mathbf{x})\right)_{\mid \mathbf{x}=\hat{\mathbf{x}}_{k \mid k-1}}^{T}=\left(-\cos \left(\theta_{k}\right)-\sin \left(\theta_{k}\right)\right)^{T}
$$

En ce qui concerne l'IEKF, la linéarisation ne se fait plus autour d'un point d'équilibre mais autour de l'élément neutre du groupe $\varphi_{x^{-1}}(x)$. Dans notre cas, l'élément neutre du groupe est équivalent au point d'équilibre de l'équation d'évolution car le modèle d'évolution est linéaire. 


\section{- Mise en évidence de l'erreur de sortie invariante sur l'IUKF :}

Les principes de l'algorithme UKF reposent sur une utilisation implicite d'une technique connue sous le nom de régression linéaire statistique pondérée (Weighted Statistical Linear Regression, en anglais WSLR). Cette technique propage les sigmas points représentatifs de la moyenne $\mathcal{X}^{(0)}=\hat{\mathbf{x}}$ et des bornes $\mathcal{X}^{(1)}=\hat{\mathbf{x}}-\sigma_{\mathbf{x}}$ et $\mathcal{X}^{(2)}=\hat{\mathbf{x}}+\sigma_{\mathbf{x}}$ où $\sigma_{\mathbf{x}}$ constitue l'écart-type de la distribution associée à $\mathbf{x}$. La propagation de ces points permet d'obtenir les sigma-points images (ou a posteriori) $\gamma_{i}$. Dès lors, il est possible de calculer l'erreur commise entre le sigma point prédit par l'état et l'état prédit par le modèle d'évolution d'une part et d'autre part l'erreur commise entre le sigma point prédit par la sortie et la sortie prédite par le modèle d'observation . Ainsi, la Figure 4 fournit à chaque pas de temps les erreurs calculées respectivement par l'UKF et l'IUKF à partir des sigmas points $\mathcal{X}^{(0)}(0,0), \mathcal{X}^{(1)}(E x m<0), \mathcal{X}^{(2)}(E x m>0)$ et de la première sortie de notre système (i.e., $y_{k}^{a_{1}}=-\sin \left(\theta_{k}\right)$ ). De plus, sont représentées différentes dynamiques d'erreur, pour deux angles particuliers $\theta=0$ ou 80 degrés à estimer. En effet, deux cas nous intéressent : soit nous disposons d'un modèle de sortie linéaire à $\theta=0$ degré, moyennant un bruit relativement faible, soit d'un modèle de sortie fortement non linéaire, à $\theta=80$ degrés. La simulation s'est donc faite avec un bruit Gaussien de matrice de covariance $\mathbf{Q}_{u}=1 e-2$ et $\mathbf{R}_{v}=0.5 \mathrm{rad}$.

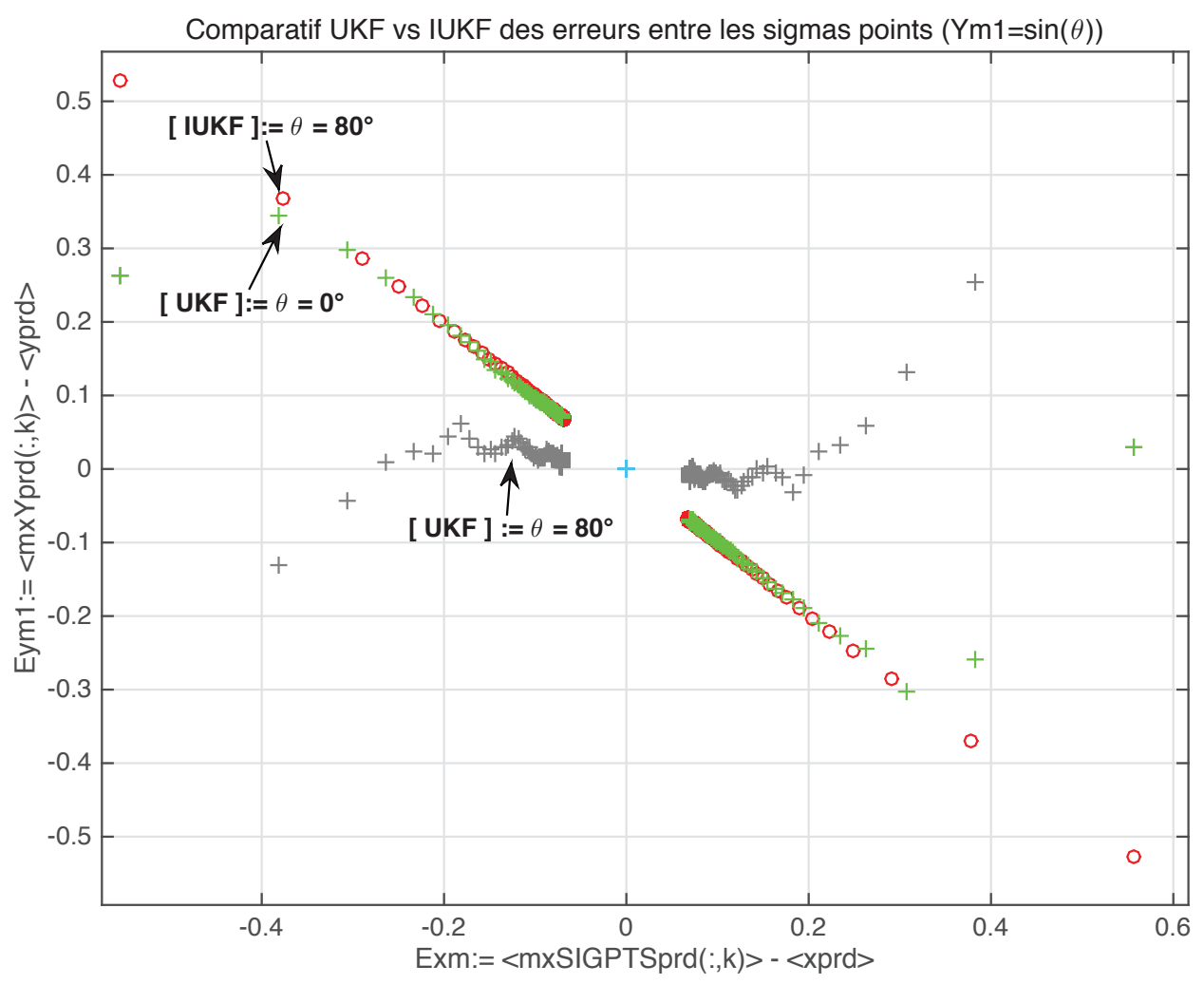

Figure 4. Comparaison des gains UKF et IUKF

Ce faisant, nous pouvons observer sur la Figure 4 que les algorithmes UKF et IUKF génèrent des erreurs, fonction de l'angle d'attitude à estimer, ne convergeant pas de la même manière. Les résultats permettent de mettre en évidence les principes théoriques discutés précédemment puisqu'il apparaît ici clairement que la convergence des erreurs calculée par l'IUKF est la même quelque soit l'angle d'attitude estimé alors que la convergence des erreurs calculée par l'UKF est différente suivant l'angle estimé par 
l'algorithme. De ce fait, les sigmas points de l'IUKF convergent systématiquement comme si l'on se trouvait constamment dans la partie linéaire du système d'observation, c'est à dire pour $\theta=0$ degré. Ce résultat illustre les propriétés d'invariance de l'IUKF puisque son comportement vis-à-vis des erreurs d'estimation est le même quelque soit l'angle d'attitude à estimer. Pour chacune des trajectoires générées à partir des différents angles d'attitude, l'erreur de sortie classique est projetée dans un repère de Frenet, qui préserve les symétries du système. De ce résultat, il faut être certain que les performances de l'IUKF restent identiques à celles de l'UKF sinon meilleures. Pour cela, nous allons analyser les performances de l'IEKF, l'IUKF, l'EKF et l'UKF à partir de la borne de Cramer-Rao.

\section{Quelques remarques sur les gains de l'IUKF :}

La Figure 5 dessine les variations temporelles de tous les gains de correction, de chaque variation d'angle d'attitude, pour l'UKF et l'IUKF. Les différentes échelles en ordonnée sont identiques entre les deux cas ce qui facilite la lecture des résultats. Une nouvelle fois, la relative constance des gains de Kalman caractérisant l'IUKF apparaît comme remarquable. Elle est l'illustration du cadre invariant introduit pour l'estimation de l'état du système. Les nombreuses et fréquentes fluctuations des gains de l'UKF pour chacun des angles sont annulées lorsque l'on passe à la version invariante du filtrage et le seul gain calculé par l'IUKF est le même que celui calculé par l'UKF à $\theta=0$ degrès.
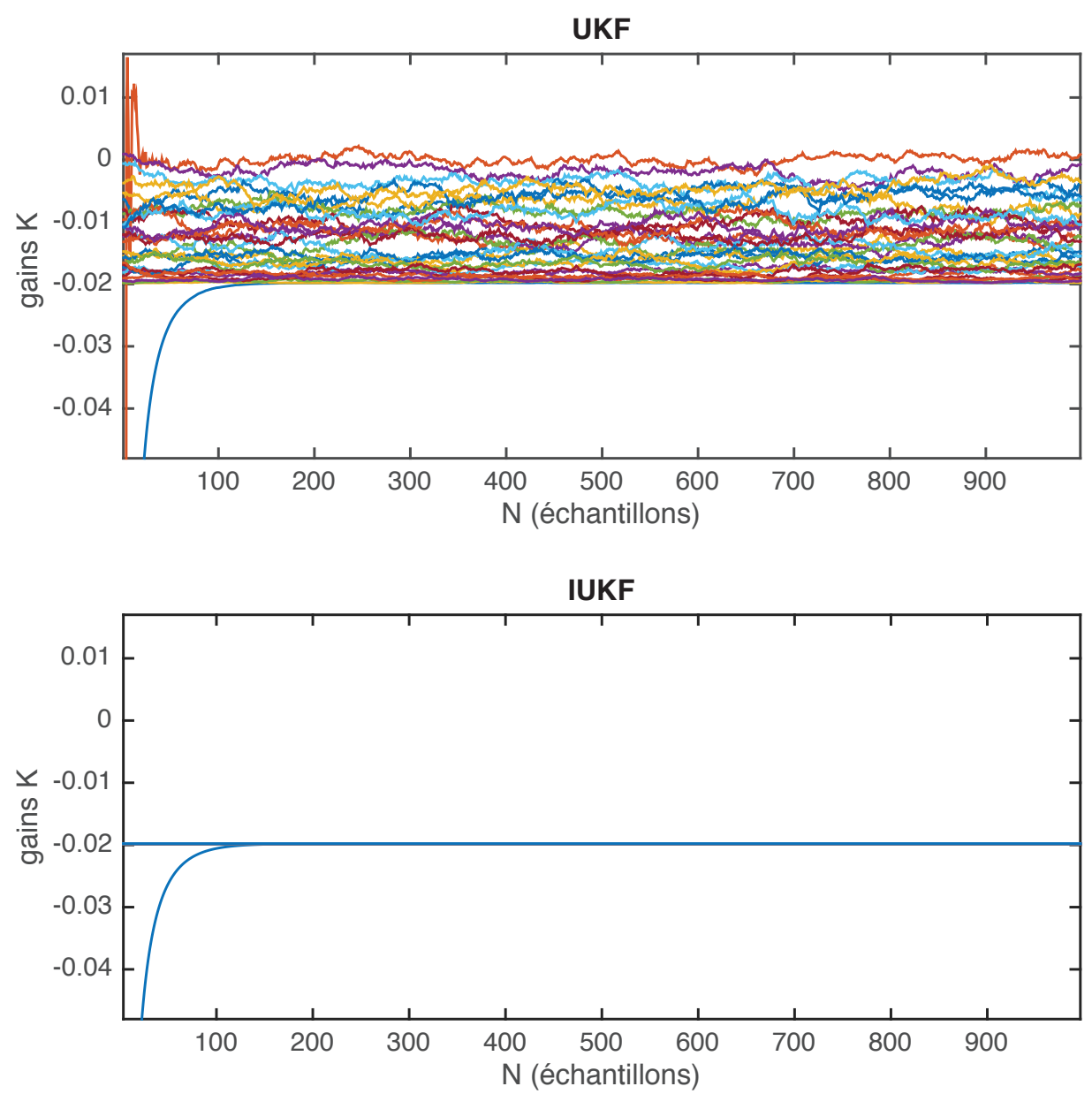

Figure 5. Variation tremporelle de tous les gains de correction, de chaque variantion de $\theta$ 


\subsection{Analyse des performances}

Dans la pratique, il est très utile de pouvoir borner (inférieurement) la variance d'un quelconque estimateur. Les Bornes de Cramer-Rao (BCR) donnent l'expression de la variance minimale que l'on peut atteindre en estimant le paramètre $\theta$. Elles sont donc particulièrement intéressantes et ce pour plusieurs raisons :

- si un estimateur atteint les bornes, et ce quel que soit $\theta$, alors c'est l'estimateur MVU (Minimum Variance Unbiaised). On sait qu'on ne pourra donc plus améliorer l'estimation.

- ces bornes sont une référence à laquelle on peut comparer tous les estimateurs. En particulier, connaître l'éloignement par rapport aux BCR est instructif parce qu'on connaît alors la "marge" qui peut encore être gagnée. Supposons que l'on possède un estimateur simple, dont la variance est 2 fois plus importante que les BCR. On peut alors se demander si le surcoût calculatoire qui sera peut être nécessaire pour construire un nouvel estimateur qui se rapprochera des BCR vaut la peine ou non.

Largement utilisée en estimation paramétrique, l'inégalité de Cramer-Rao est aussi définie pour les processus aléatoires. Cette borne donne donc une indication sur les limites de performance de l'estimateur.

L'énoncé de l'inégalité de Cramer-Rao est le suivant : soit un estimateur $\hat{x}_{n \mid n}$ d'un vecteur aléatoire $x_{n}$ construit à partir d'un processus observé $y_{k}, k \in\{0, \cdots, n\}$. On suppose que la FDP (Fonction de Densité de Probabilité) $p_{x_{n}}(x)$ vérifient la condition de régularité suivante :

$$
\lim _{x \rightarrow \infty} B(x) p_{x_{n}}(x)=0 \text { avec } B(x)=\int_{-\infty}^{+\infty}\left(\hat{x}_{n \mid n}-x\right) p\left(y_{0}, y_{1}, \cdots, y_{n} \mid x n=x\right) d y_{1} \cdots d y_{n}
$$

A noter que $B(x)$ fait partie d'une sous-classe de processus stochastique appelé processus markovien. Alors, quel que soit l'estimateur non biaisé $\hat{x}$, la variance et la matrice de covariance de $\hat{x}_{n \mid n}$ vérifie l'inégalité suivante :

$$
E\left[\left(\hat{x}_{n \mid n}-x_{n}\right)\left(\hat{x}_{n \mid n}-x_{n}\right)^{\prime}\right] \geqslant I^{-1}\left(x_{n}\right), \text { ou } I\left(x_{n}\right)=-E\left[\frac{\partial}{\partial x_{n}}\left(\frac{\partial}{\partial x_{n}} \ln p\left(y_{n}, x_{n}\right)\right)^{\prime}\right]
$$

Avec $\frac{\partial}{\partial x_{n}}$ opérateur dérivé partiel par rapport à $x_{n}$ et $I(n)$ est l'information de Fisher de $x_{n}$. La séquence $I\left(x_{n}\right)$ s'obtient à l'aide de l'expression récursive :

$$
\begin{aligned}
& I\left(x_{n-1}\right)=D_{n}^{22}-D_{n}^{21}\left(I\left(x_{n}\right)+D_{n}^{11}\right)^{-1} D_{n}^{12} \\
& \operatorname{avec}\left\{\begin{array}{l}
D_{n}^{11}=-E\left[\frac{\partial}{\partial x_{n}}\left(\frac{\partial}{\partial x_{n}} \ln p\left(x_{n-1} \mid x_{n}\right)\right)^{\prime}\right] \\
D_{n}^{12}=-E\left[\frac{\partial}{\partial x_{n}}\left(\frac{\partial}{\partial x_{n+1}} \ln p\left(x_{n-1} \mid x_{n}\right)\right)^{\prime}\right]=D_{n}^{21^{\prime}} \\
D_{n}^{22}=-E\left[\frac{\partial}{\partial x_{n+1}}\left(\frac{\partial}{\partial x_{n+1}} \ln p\left(x_{n-1} \mid x_{n}\right)\right)^{\prime}\right]-E\left[\frac{\partial}{\partial x_{n+1}}\left(\frac{\partial}{\partial x_{n+1}} \ln p\left(y_{n-1} \mid x_{n+1}\right)\right)^{\prime}\right] \\
I\left(x_{0}\right)=-E\left[\frac{\partial}{\partial x_{0}}\left(\frac{\partial}{\partial x_{0}} \ln p\left(x_{0}\right)\right)^{\prime}\right]
\end{array}\right.
\end{aligned}
$$


La BCR s'obtient à partir de la matrice d'information de Fisher $I\left(x_{n}\right)$ tel que $\mathrm{BCR}=I\left(x_{n}\right)^{-1}$. Une des particularités de cette information est qu'un estimateur est dit efficace, si et seulement si, il atteint la $\mathrm{BCR}$ quelque soit l'état à estimer. Cette borne est atteignable uniquement dans le cas où le système est linéaire. Dès lors, les principes théoriques de la première section vont nous être très utiles. Ils ont permis de discuter de la dynamique de l'erreur d'estimation sur l'état invariante. Celle-ci obéit dans certain cas à une équation d'erreur dite autonome et donc permet de se ramener au cas d'un système au comportement linéaire sur l'erreur d'état. Ainsi, dans (Bonnabel [2007]) S.Bonnabel et A. Barrau ont démontré que pour un système paramétré par un groupe $S O(3)$, la borne de Cramér-Rao coincide parfaitement avec la covariance retournée par l'algorithme IEKF. Dans notre cas, l'équation d'évolution est linéaire. Ainsi, nous devons retrouver la même covariance sur l'état, que l'algorithme soit invariant ou non. Néanmoins, les résultats précédents nous ont montré que lorsque le modèle d'observation était non-linéaire, le filtre de Kalman invariant permettait d'obtenir grâce à l'erreur de sortie invariante, un comportement d'erreur de sortie linéaire. Reprenons les équations du modèle d'évolution [2] et d'observation [1] et calculons l'information de Fisher à partir de l'équation récursive [9]. On a :

$$
\left\{\begin{array}{l}
p\left(x_{n+1} \mid x_{n}\right)=\frac{1}{\sqrt{2 \pi Q_{u}}} \exp \left\{\frac{-1}{2 Q_{u}}\left(x_{n-1}-x_{n}\right)^{2}\right\} \\
p\left(y_{n+1} \mid x_{n+1}\right)=\frac{1}{\sqrt{2 \pi R_{v}}} \exp \left\{\frac{-1}{2 R_{u}}\left(y_{n+1}^{a_{1}}+\sin \left(x_{n+1}\right)\right)^{2}+\left(y_{n+1}^{a_{3}}-\cos \left(x_{n+1}\right)\right)^{2}\right\}
\end{array}\right.
$$

On déduit de ces équations $D_{n}^{11}, D_{n}^{12}, D_{n}^{21}$ et $D_{n}^{22}$ :

$$
\left\{\begin{array}{l}
D_{n}^{11}=-E\left[\frac{\partial}{\partial x_{n}}\left(\frac{\partial}{\partial x_{n}} \ln p\left(x_{n-1} \mid x_{n}\right)\right)^{\prime}\right]=\frac{1}{Q_{u}} \\
D_{n}^{12}=-E\left[\frac{\partial}{\partial x_{n}}\left(\frac{\partial}{\partial x_{n+1}} \ln p\left(x_{n-1} \mid x_{n}\right)\right)^{\prime}\right]=\frac{-1}{Q_{u}}=D_{n}^{21^{\prime}} \\
D_{n}^{22}=-E\left[\frac{\partial}{\partial x_{n+1}}\left(\frac{\partial}{\partial x_{n+1}} \ln p\left(x_{n-1} \mid x_{n}\right)\right)^{\prime}\right]-E\left[\frac{\partial}{\partial x_{n+1}}\left(\frac{\partial}{\partial x_{n+1}} \ln p\left(y_{n-1} \mid x_{n+1}\right)\right)^{\prime}\right]=\frac{1}{Q_{u}}+\frac{1}{R_{v}}
\end{array}\right.
$$

L'équation [9] s'écrit alors

$$
I\left(x_{n+1}\right)=\frac{1}{R_{v}}+\frac{I\left(x_{n}\right)}{I\left(x_{n}\right) Q_{u}+1}
$$

Sur la Figure 6 sont représentées les trajectoires de l'écart-type de l'erreur d'estimation de l'état par les filtres EKF, UKF, IEKF, IUKF ainsi que la borne de Cramer-Rao (BCR) à posteriori pour une covariance de bruit de mesure égale à 0.5 et une covariance de bruit de l'état égale à $1 e-2$. Comme prévu, l'écarttype de l'erreur d'estimation de l'état pour l'EKF est la même que celle calculée par l'IEKF. Il en est de même pour les algorithmes UKF et IUKF. Pour ces algorithmes, l'écart-type de l'erreur d'estimation est comme attendu, supérieur à la BCR malgré que celui calculé par l'IUKF est très proche de la BCR. Ce résultat nous permet d'en déduire que l'algorithme IUKF, tout comme l'algorithme UKF, a d'excellentes performances qui ne sont en rien dégradées par l'invariance introduite dans le filtre UKF. Afin de ne pas alourdir cet article, les réponses temporelles de chaque filtre n'apparaissent pas. 


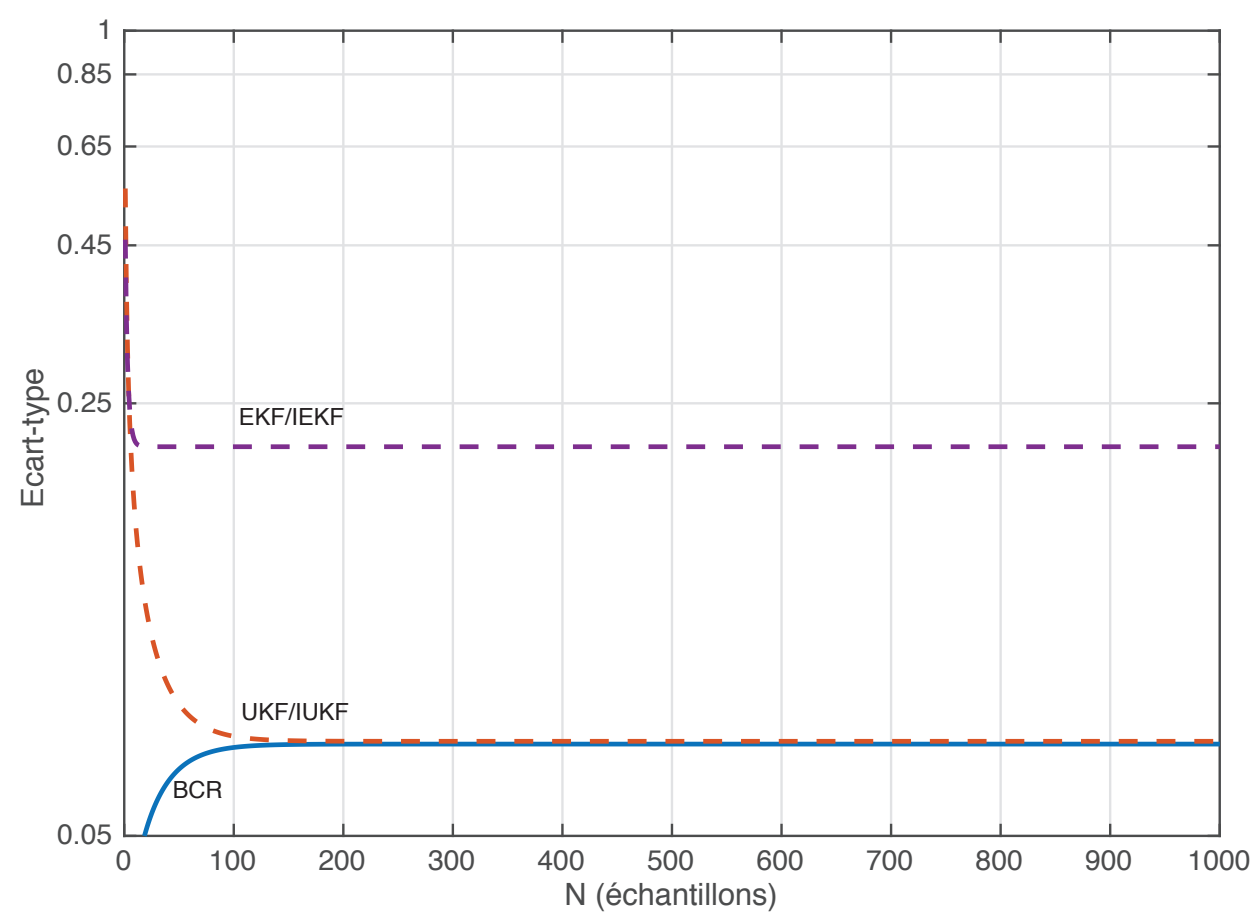

Figure 6. Ecart-type de l'erreur d'estimation de l'état par les filtres et BCR

\section{Illustration de l'IUKF pour le cas d'un INS (Inertial Navigation System)}

Nous allons maintenant utiliser l'algorithme IUKF pour résoudre la problématique d'estimation propre à l'INS. Pour ce faire, nous devons formuler un observateur invariant construit à partir des équations d'évolution et d'observation de l'INS. Dans ce problème, les mesures de vitesse et de position délivrées par un GPS (Global Positioning System) et un baromètre viennent enrichir les mesures disponibles, en plus de celles déjà fournies par les accéléromètres et les magnétomètres. Grâce aux invariances galiléennes, les équations du modèle peuvent être exprimées indifféremment dans le repère avion ou dans le repère terrestre. Les équations du modèle de l'INS (Condomines et al. [2013]) rappelées ci-contre correspondent à la description de la dynamique de l'engin et des mesures dans le repère terrestre avec $q$ le quaternion de norme unité. La représentation d'état non linéaire correspondante au modèle $\mathcal{M}_{\text {ins }}^{+}$peut-être décrite sous une forme compacte telle que $\dot{\mathbf{x}}=f(\mathbf{x}, \mathbf{u})$ et $\mathbf{y}=h(\mathbf{x}, \mathbf{u})$ où $\mathbf{x}=\left(q^{T} V^{T} X^{T} \omega_{b}^{T} a_{s} h_{b}\right)^{T}, \mathbf{u}=\left(\omega_{m}^{T} a_{m}^{T}\right)^{T}$ et $\mathbf{y}=\left(y_{V}^{T} y_{X}^{T} y_{h} y_{B}^{T}\right)^{T}$. Pour rappel :

$$
\mathcal{M}_{i n s}^{+}\left\{\begin{array}{l}
\dot{q}=\frac{1}{2} q *\left(\omega_{m}-\omega_{b}\right) \\
\dot{V}=A+\frac{1}{a_{s}} q * a_{m} * q^{-1} \\
\dot{X}=V \\
\dot{\omega}_{b}=0 \\
\dot{a}_{s}=0 \\
\dot{h}_{b}=0
\end{array} \quad \text { (évolution) } \quad\left(\begin{array}{l}
y_{V} \\
y_{X} \\
y_{h} \\
y_{B}
\end{array}\right)=\left(\begin{array}{c}
V \\
X \\
X_{z}-h_{b} \\
q^{-1} * B * q
\end{array}\right) \quad\right. \text { (observation) }
$$

En considérant les équations du modèle $\mathcal{M}_{\text {ins }}^{+}$et le groupe de Lie $G=\mathbb{H}_{1} \times \mathbb{R}^{11}$ agissant sur tout l'espace d'état associé à la description de la dynamique du système, les transformations locales de groupe 
suivantes permettent de montrer que la modélisation INS est $G$-invariante et $G$-équivariante. Ainsi $\forall g=$ $\left(q_{0}^{T} V_{0}^{T} X_{0}^{T} \omega_{0}^{T} a_{0} h_{0}\right)^{T} \in G:$

$$
\varphi_{g}(\mathbf{x})=\left(\begin{array}{c}
q * q_{0} \\
V+V_{0} \\
X+X_{0} \\
q_{0}^{-1} * \omega_{b} * q_{0}+\omega_{0} \\
a_{s} \cdot a_{0} \\
h_{b}+h_{0}
\end{array}\right), \psi_{g}(\mathbf{u})=\left(\begin{array}{c}
q_{0}^{-1} * \omega_{m} * q_{0}+\omega_{0} \\
a_{0} \cdot q_{0}^{-1} * a_{m} * q_{0}
\end{array}\right), \rho_{g}(\mathbf{y})=\left(\begin{array}{c}
y_{V}+V_{0} \\
y_{X}+X_{0} \\
y_{h}-h_{0}+<X_{0}, e_{3}> \\
q_{0}^{-1} * y_{B} * q_{0}
\end{array}\right)
$$

Ces transformations sont équivalentes soit à une rotation, soit à une translation, soit à la composition d'une rotation et d'une translation qui ré-expriment les quantités vectorielles $\mathbf{x}, \mathbf{u}$ et $\mathbf{y}$ dans le repère terrestre ou engin. La recherche des invariants du problème, par la méthode du repère mobile, vise à déterminer le paramétrage du groupe $G$ qui envoie le vecteur d'état x vers l'élément neutre. Si nous considérons le cas particulier $g=\mathbf{x}^{-1}=\left(\left(q^{-1}\right)^{T}-V^{T}-X^{T}-\left(q * \omega_{b} * q^{-1}\right)^{T} a_{s}^{-1}-h_{b}+<X, e_{3}>\right)^{T}$, nous obtenons :

$$
\varphi_{\mathbf{x}^{-1}}(\mathbf{x})=\left(\begin{array}{c}
q * q^{-1} \\
V-V \\
X-X \\
q * \omega_{b} * q^{-1}-q * \omega_{b} * q^{-1} \\
a_{s} \cdot a_{s}^{-1} \\
h_{b}+<X, e_{3}>-\left(h_{b}+<X, e_{3}>\right)
\end{array}\right)=\left(\begin{array}{l}
1 \\
\overrightarrow{0} \\
\overrightarrow{0} \\
\overrightarrow{0} \\
1 \\
0
\end{array}\right)=e
$$

\subsection{Formulation de l'observateur invariant pour l'INS}

En suivant la méthodologie présentée dans Condomines et al. [2013], une structure possible pour tout observateur invariant, peut être explicitée ici dans le cas de l'INS comme il suit :

$\mathcal{O}_{\mathcal{M}_{i n s}^{+}}\left\{\begin{array}{l}\dot{\hat{q}}=\frac{1}{2} \hat{q} *\left(\omega_{m}-\hat{\omega}_{b}\right)+\sum_{i=1}^{3}\left(\sum_{j=1}^{3}\left(\overline{\mathbf{K}}_{V \rightarrow q}^{(i j)} \mathrm{E}_{V}+\overline{\mathbf{K}}_{X \rightarrow q}^{(i j)} \mathrm{E}_{X}+\overline{\mathbf{K}}_{B \rightarrow q}^{(i j)} \mathrm{E}_{B}\right)+\overline{\mathbf{K}}_{h \rightarrow q}^{(i j)} \mathrm{E}_{h}\right) \cdot e_{i} * \hat{q} \\ \dot{\hat{V}}=A+\frac{\hat{q} * a_{m} * \hat{q}^{-1}}{\hat{a}_{s}}+\sum_{i=1}^{3}\left(\sum_{j=1}^{3}\left(\overline{\mathbf{K}}_{V \rightarrow V}^{(i j)} \mathrm{E}_{V}+\overline{\mathbf{K}}_{X \rightarrow V}^{(i j)} \mathrm{E}_{X}+\overline{\mathbf{K}}_{B \rightarrow V}^{(i j)} \mathrm{E}_{B}\right)+\overline{\mathbf{K}}_{h \rightarrow V}^{(i j)} \mathrm{E}_{h}\right) \cdot e_{i} \\ \dot{\hat{X}}=\hat{V}+\sum_{i=1}^{3}\left(\sum_{j=1}^{3}\left(\overline{\mathbf{K}}_{V \rightarrow X}^{(i j)} \mathrm{E}_{V}+\overline{\mathbf{K}}_{X \rightarrow X}^{(i j)} \mathrm{E}_{X}+\overline{\mathbf{K}}_{B \rightarrow X}^{(i j)} \mathrm{E}_{B}\right)+\overline{\mathbf{K}}_{h \rightarrow X}^{(i j)} \mathrm{E}_{h}\right) \cdot e_{i} \\ \dot{\hat{\omega}}_{b}=\hat{q}^{-1} * \sum_{i=1}^{3}\left(\sum_{j=1}^{3}\left(\overline{\mathbf{K}}_{V \rightarrow \omega_{b}}^{(i j)} \mathrm{E}_{V}+\overline{\mathbf{K}}_{X \rightarrow \omega_{b}}^{(i j)} \mathrm{E}_{X}+\overline{\mathbf{K}}_{B \rightarrow \omega_{b}}^{(i j)} \mathrm{E}_{B}\right)+\overline{\mathbf{K}}_{h \rightarrow \omega_{b}}^{(i j)} \mathrm{E}_{h}\right) \cdot e_{i} * \hat{q} \\ \dot{\hat{a}}_{s}=\hat{a}_{s} \cdot\left(\sum_{j=1}^{3}\left(\overline{\mathbf{K}}_{V \rightarrow a_{s}}^{(j)} \mathrm{E}_{V}+\overline{\mathbf{K}}_{X \rightarrow a_{s}}^{(j)} \mathrm{E}_{X}+\overline{\mathbf{K}}_{B \rightarrow a_{s}}^{(j)} \mathrm{E}_{B}\right)+\overline{\mathbf{K}}_{h \rightarrow a_{s}}^{(j)} \mathrm{E}_{h}\right) \\ \dot{\hat{h}}_{b}=\sum_{j=1}^{3}\left(\overline{\mathbf{K}}_{V \rightarrow b_{s}}^{(j)} \mathrm{E}_{V}+\overline{\mathbf{K}}_{X \rightarrow b_{s}}^{(j)} \mathrm{E}_{X}+\overline{\mathbf{K}}_{B \rightarrow b_{s}}^{(j)} \mathrm{E}_{B}\right)+\overline{\mathbf{K}}_{h \rightarrow b_{s}}^{(j)} \mathrm{E}_{h}\end{array}\right.$ 
où l'expression de l'erreur de sortie invariante $E$ est donnée par :

$$
\left(\begin{array}{c}
\mathbf{E}_{V} \\
\mathbf{E}_{X} \\
\mathbf{E}_{B} \\
\mathbf{E}_{h}
\end{array}\right)=h\left(e=\varphi_{\hat{\mathbf{x}}^{-1}}(\hat{\mathbf{x}}), \psi_{\hat{\mathbf{x}}^{-1}}(\mathbf{u})\right)-\rho_{\hat{\mathbf{x}}^{-1}}(\mathbf{y})=\left(\begin{array}{c}
\hat{V}-y_{v} \\
\hat{X}-y_{x} \\
<\hat{X}, e_{3}>-\hat{h}_{b}-y_{h} \\
B-\hat{q} * y_{B} * \hat{q}^{-1}
\end{array}\right)
$$

Dans les équations du filtre, nous retrouvons les composantes du repère invariant en bleu et les différents gains matriciels $\overline{\mathbf{K}}$ en vert. Les valeurs de ces matrices de gain peuvent être fixées arbitrairement pour imposer une dynamique souhaitée à l'estimateur. En effet, dans le cas de la synthèse d'observateurs invariants selon les principes de la méthode constructive, appliquée ici aux cas de l'INS, il est possible de dériver un ensemble de relations analytiques qui permettent de déduire directement, et donc analytiquement, des valeurs admissibles pour les gains. C'est en cela que l'approche issue de la littérature du domaine porte le qualificatif de constructive. Il en résulte immédiatement que la convergence des erreurs d'estimation peut être assurée (au sens des invariants du problème d'estimation) et que, conjointement, la dynamique de l'estimateur peut être choisie librement. Pour pallier à la difficulté de régler les gains de correction manuellement et de s'affranchir de la linéarisation de l'erreur d'estimation sur l'état invariant, nous allons appliquer le filtre IUKF. On en déduit l'expression analytique $\eta(\mathbf{x}, \hat{\mathbf{x}})$ telle que :

$$
\eta(\mathbf{x}, \hat{\mathbf{x}})=e-\varphi_{\mathbf{x}^{-1}}(\hat{\mathbf{x}})=\left(\begin{array}{l}
\mathbf{1} \\
0 \\
0 \\
0 \\
1 \\
0
\end{array}\right)-\left(\begin{array}{c}
\hat{q} * q^{-1} \\
\hat{V}-V \\
\hat{X}-X \\
q *\left(\hat{w}_{b}-w_{b}\right) * q^{-1} \\
\hat{a}_{s} / a_{s} \\
\hat{h}_{b}-h_{b}
\end{array}\right)=\left(\begin{array}{c}
\mathbf{1}-\hat{q} * q^{-1} \\
V-\hat{V} \\
X-\hat{X} \\
q *\left(w_{b}-\hat{w}_{b}\right) * q^{-1} \\
1-\hat{a}_{s} / a_{s} \\
h_{b}-\hat{h}_{b}
\end{array}\right)
$$

\subsection{Premiers résultats et analyse du RIUKF}

Les données d'entrée ayant servi de référence pour cette première évaluation de l'approche de type RIUKF ${ }^{3}$ sur un INS correspondent à celles issues d'une simulation d'un modèle dynamique décrivant le mouvement de chute libre d'un parafoil (https ://flightopportunities.nasa.gov/technologies/66/). Ces données simulées, traitées sans bruit, ont permis de valider facilement les principes méthodologiques développés par JP.Condomines, C. Seren et G.Hattenberger dans le cas d'un AHRS et de tirer les premières conclusions pour le RIUKF appliquée à un INS. L'horizon temporel de la simulation de référence sélectionnée pour valider l'algorithme est d'un peu plus de 100 secondes. La dynamique simulée du système de parachute est relativement forte comme l'illustreront

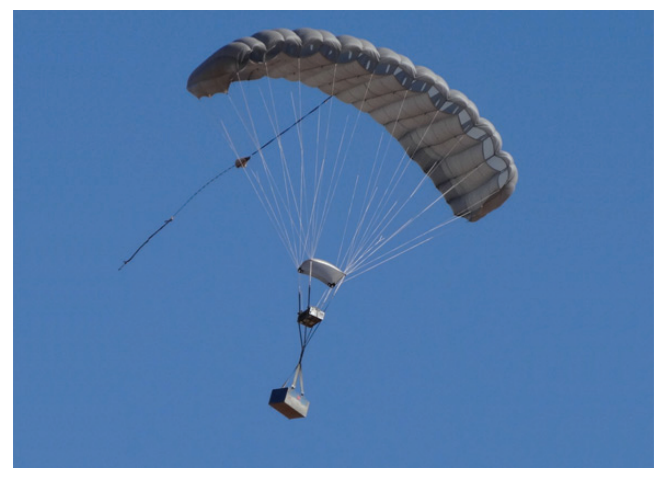

Figure 7. Modèle de parafoil développé par la NASA avec sa charge utile les figures retraçant les évolutions temporelles de l'attitude, de la vitesse et de la position de référence

3.La multiplication à droite du quaternion $q$ par le quaternion $q_{0}$ effectuée dans la section précédente justifie le terme Right IUKF dans la suite du document. 
de l'engin. En effet, nous y observerons des variations d'angle de gîte, de cap et d'assiette de plusieurs dizaines de degrés ainsi que des variations de vitesse de plusieurs dizaines de mètres par seconde.

Enfin, les résultats et estimations obtenus en appliquant l'algorithme du RIUKF aux données sont systématiquement comparés avec ceux fournis par un algorithme UKF standard.

\section{- Estimation de l'attitude :}

La figure 8 fournit respectivement les estimés UKF et RIUKF des angles d'attitude de l'engin, et les comparent dans les deux cas aux pseudo-mesures associées qui ont été reconstruites à partir de la donnée des composantes du vecteur d'état servant de référence correspondantes au quaternion du système. Les angles estimés collent quasi-parfaitement dans les deux cas aux valeurs de référence. Comme dans le cas de l'AHRS (Attitude and Heading Reference System) présenté dans, que cela soit pour l'UKF ou le RIUKF, l'attitude du parafoil selon les trois axes est très correctement restituée. Les tracés des erreurs d'estimation (selon une échelle logarithmique en ordonnée) montrent que l'estimateur IUKF converge mieux et plus rapidement vers les vraies valeurs des paramètres de vol. En effet, les amplitudes de ces erreurs sont plus faibles dans le cas du RIUKF. Par ailleurs, l'analyse comparée de ces graphes d'erreurs tend à prouver que les résidus d'estimation sur l'état sont plus stables au cours du temps dans le cas du RIUKF, étant donné qu'il est possible d'observer une lente et légère décroissance de ceux-ci dans le cas de l'UKF. Les estimés délivrés par l'approche RIUKF semblent donc être de meilleure qualité au regard de ceux produits par un algorithme d'estimation de type UKF standard.

\section{- Estimation de l'état associé au quaternion :}

Ayant observé une reconstitution quasi-parfaite des angles d'attitude, il apparaît normal de constater qu'il en va de même pour les composantes du quaternion (cf. Figure 10 et 11). Que ce soit pour l'UKF ou le RIUKF, le quaternion estimé est semblable en tout point à la référence. Le fait notable concerne ici les courbes à \pm 1 écart-type (théorique i.e. calculé par l'algorithme) tracées en pointillés noirs pour chaque composante de l'état associé au quaternion. Les figures 10 et 11 illustrent très clairement des différences entre l'approche de type UKF et RIUKF. En effet, pour un même réglage des matrices de covariance estimées pour les bruits d'évolution et de mesure ( $\mathbf{Q}$ et $\mathbf{R})$, le niveau d'incertitude déterminé par l'UKF apparaît très fluctuant sur les composantes $q_{1}$ et $q_{2}$ dans l'intervalle [20;30]. A l'opposé, les écarts-types théoriques calculés par l'IUKF, après un régime transitoire de quelques secondes, convergent vers des valeurs constantes autour de chaque état moyen $\left(\hat{q}_{0}, \hat{q}_{1}, \hat{q}_{2}, \hat{q}_{3} \mid \mathbf{z}\right)$, étant données les observations z. Du coup, les effets liés à la non-exactitude du modèle de l'INS utilisé se font ici ressentir dans les tracés de ces écarts-types de l'UKF et non pas dans les tracés du RIUKF.

\section{- Estimation des vitesses, des positions, des imperfections de mesure et des covariances :}

Contrairement au cas de l'AHRS, le modèle INS décrit dans la section précédente, permet d'estimer en plus des angles d'attitude, les vitesses et les positions de la chute libre du parafoil. Nous constatons que, quelque soit l'algorithme considéré, les vraies valeurs des différentes grandeurs sont estimées sans erreur. Les figures 13,14, 15 et 16 montrent les résultats obtenus pour l'UKF et le RIUKF. Un jeu de trois biais additifs (état : $\left.\omega_{b}=\left(\begin{array}{lll}0 & 0 & 0\end{array}\right)^{T}\right)$ et de deux facteurs d'échelle (états : $\left.\left(a_{s}, h_{s}\right)=(1,0)\right)$ ont 

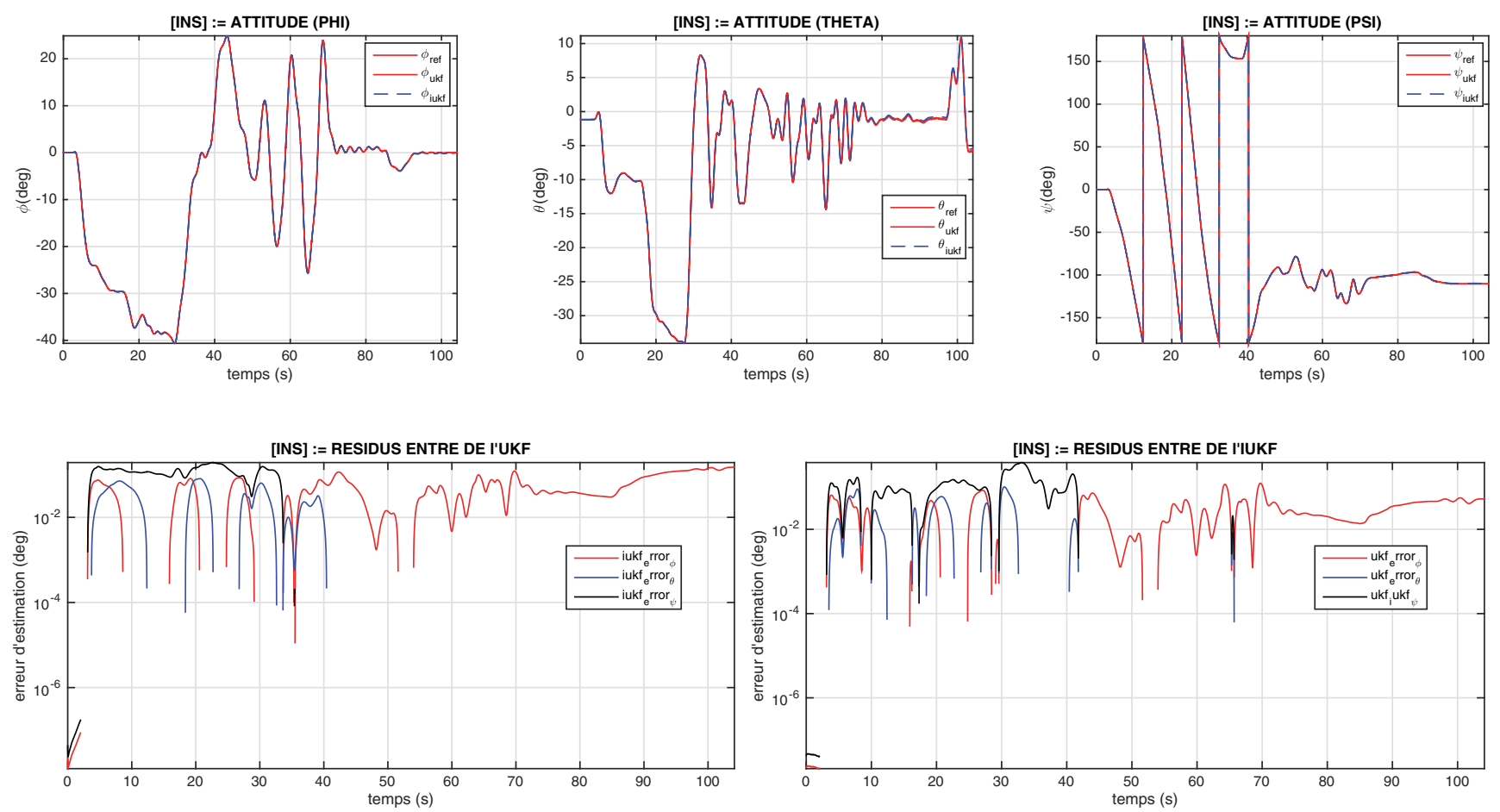

Figure 8. INS : comparaison UKF/IUKF de l'estimation d'attitude $(\phi, \theta, \psi)$ et des erreurs d'estimation
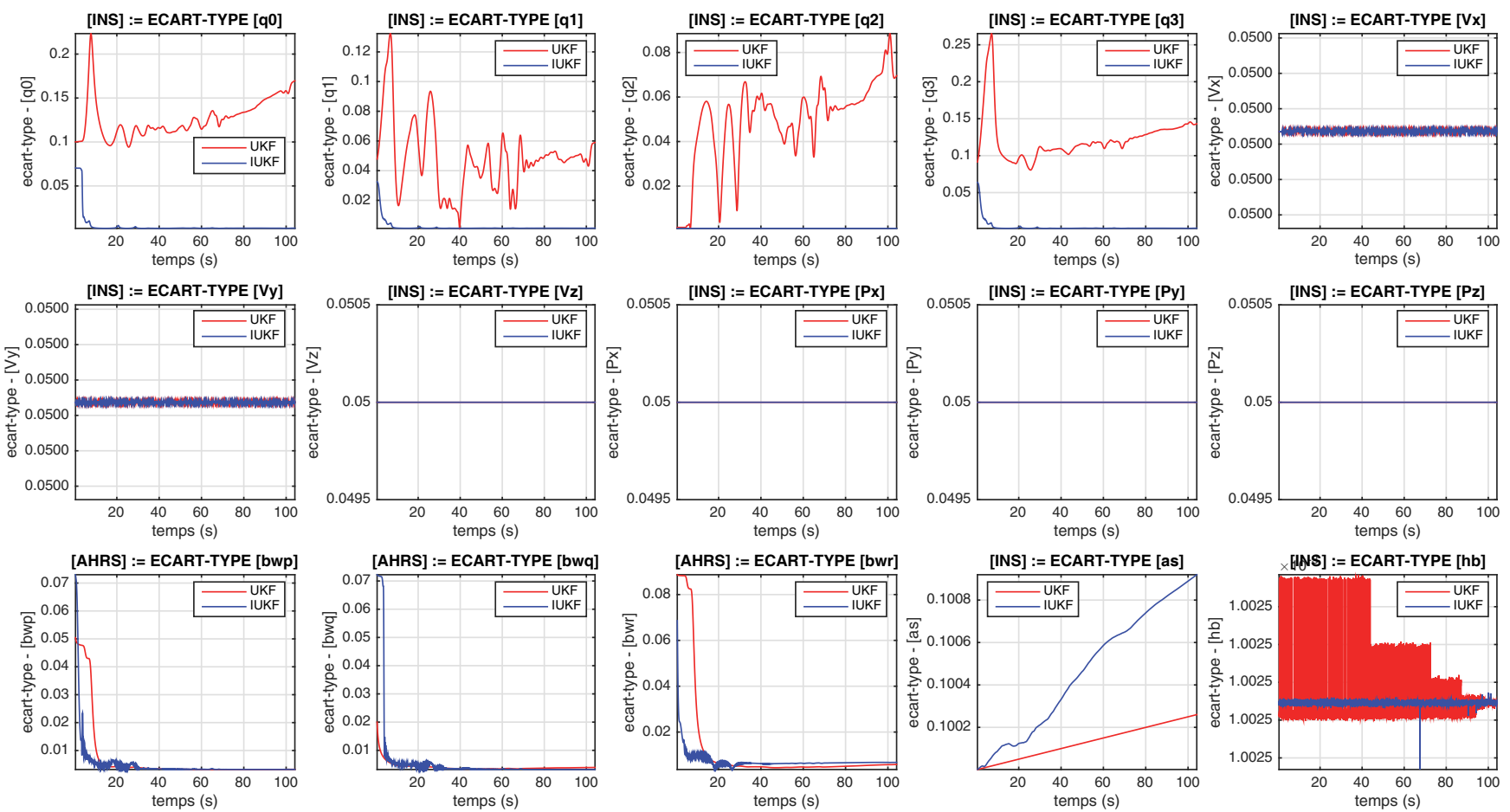

Figure 9. INS : comparaison des écarts-types théoriques calculés par l'UKF et l'IUKF

été introduits pour polluer les données simulées qui servent d'entrées aux deux schémas d'estimation évalués dans cette section. Ce faisant, l'observabilité du problème d'estimation posé peut être vérifiée et la capacité de l'algorithme employée à estimer de telles imperfections testées. Les figures 17 et 18 concatènent les résultats obtenus pour l'UKF et l'IUKF. Nous constatons que, quelque soit l'algorithme considéré, les vraies valeurs des différentes imperfections sont estimées sans erreur. Les écarts-types 

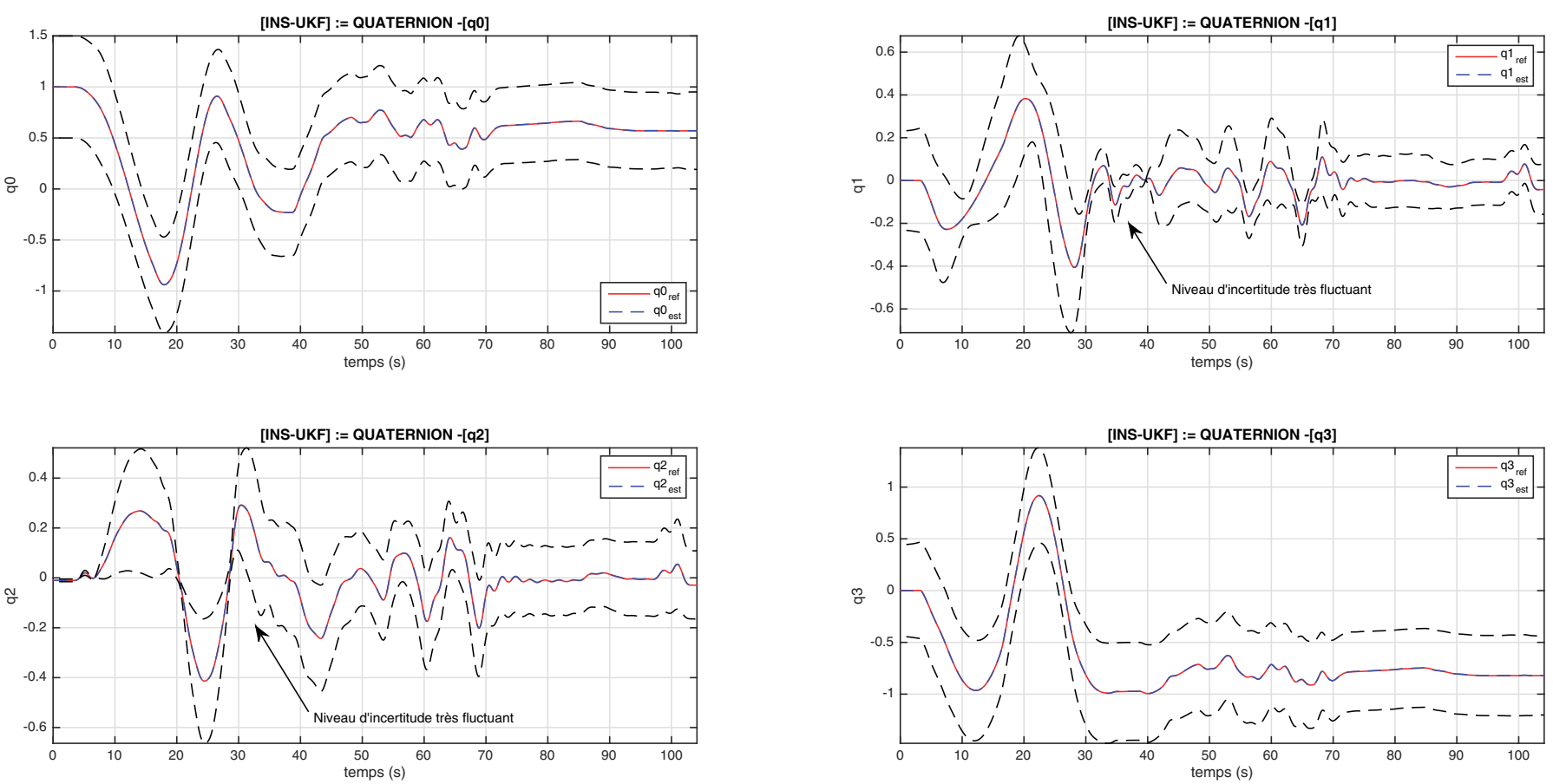

Figure 10. INS-UKF : estimation des états associés au quaternion q
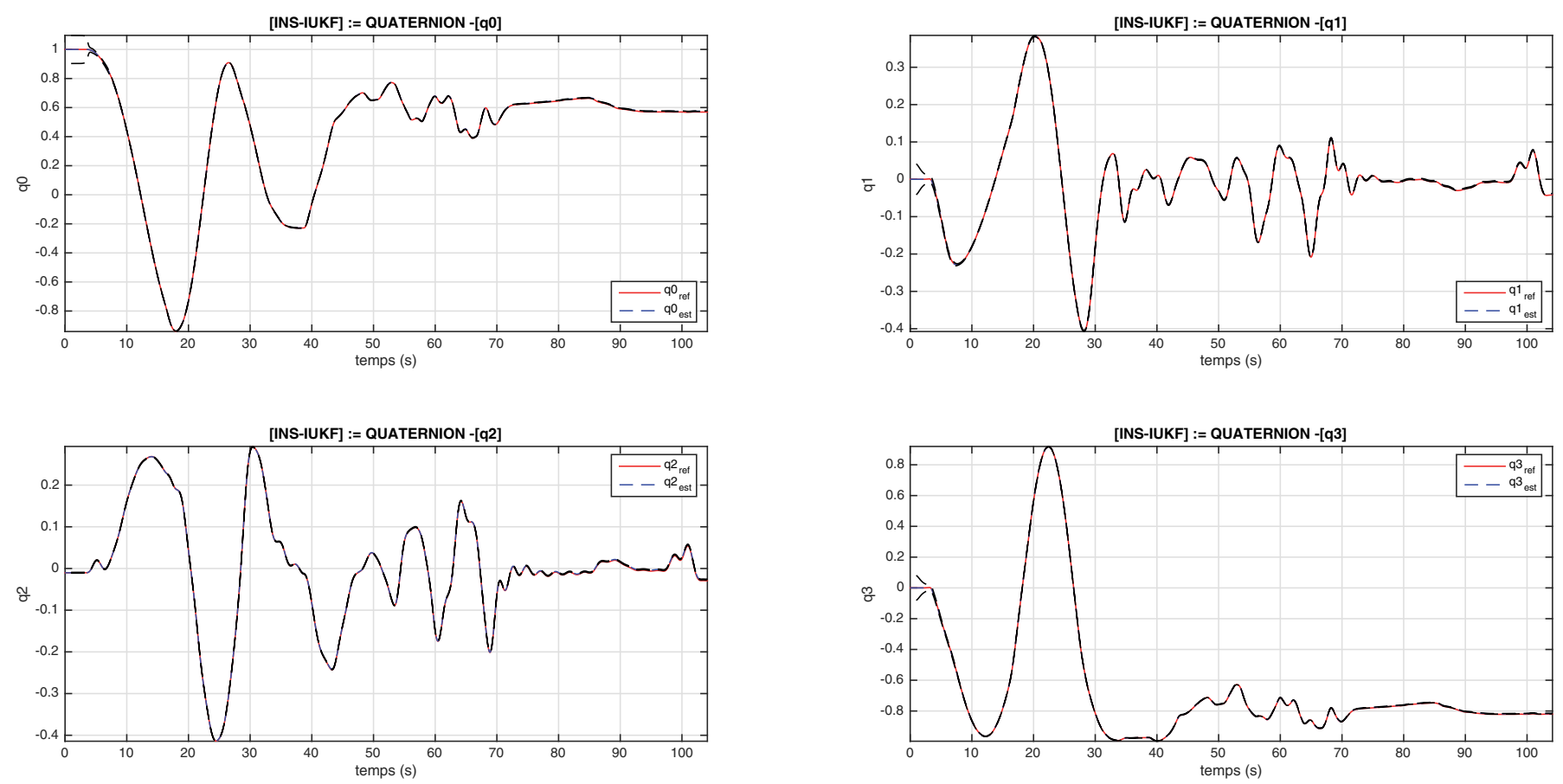

Figure 11. INS-IUKF : estimation des états associés au quaternion q

théoriques calculés par les deux algorithmes sont par ailleurs quasi-identiques entre l'UKF et sa version invariante. Nous relèverons au regard de ces différents tracés que certains effets parasites, induits par l'inexactitude de la modélisation mise en œuvre pour le traitement des données, peuvent aussi s'observer sur les écarts-types théoriques calculés par le RIUKF et associés aux états liés aux biais des gyromètres $\omega_{b}=\left(b \omega_{p}, b \omega_{q}, b \omega_{r}\right)$. Toutefois, ces effets apparaissent négligeables puisqu'ils engendrent d'infimes variations des écarts-types estimés au cours du temps. La figure 9 compare les écarts-types théoriques déterminés par l'UKF d'une part et l'IUKF d'autre part pour toutes les composantes du vecteur d'état 


\section{Conclusion}

Cette étude nous a permis de valider les principes théoriques discutés précédemment puisqu'il apparaît ici clairement que les propriétés des observateurs invariants sont vérifiées par l'IUKF dans le cas de l'INS, tout comme dans (Condomines [2015]). La constance des écarts-types théoriques, et donc des covariances estimées, sur la trajectoire définie par $I=\psi_{\hat{\mathbf{x}}^{-1}}(u)$ est illustrative du caractère invariant du schéma d'estimation mis au point. Ainsi, l'IEKF de la littérature dispose bien d'un «équivalent » fondé sur les principes de la SUT, l'IUKF, comme il a été montré jadis que l'EKF disposait d'un «équivalent» reposant sur ces mêmes principes, l’UKF (cf. Figure 12).

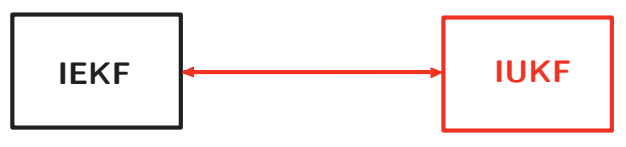

cadre invariant

cadre non invariant



Figure 12. Nouvelle formulation de l'UKF dans un cadre invariant
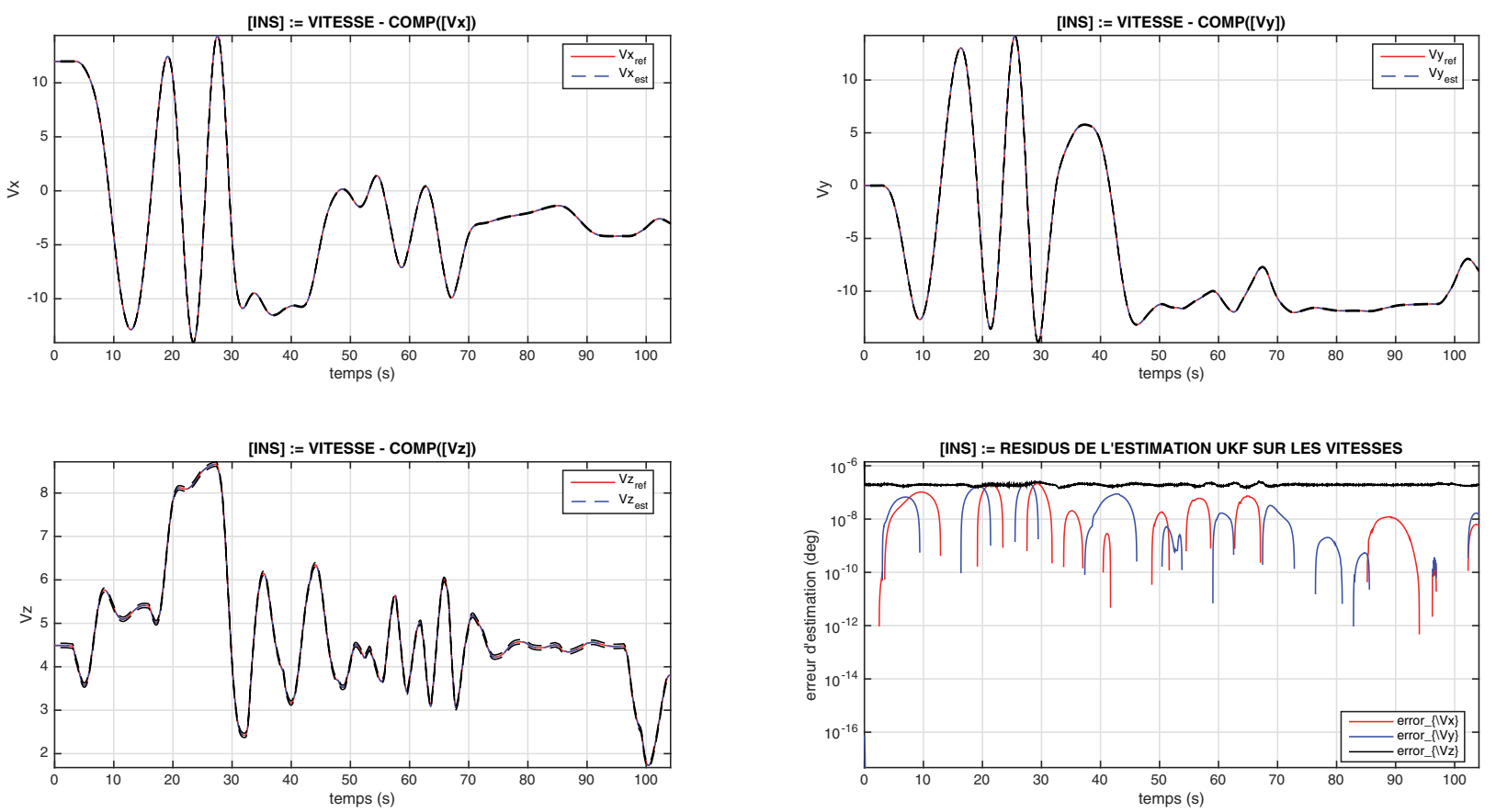

Figure 13. INS-UKF : estimation des vitesses et erreurs d'estimation

Ce qui a donc été établi pour le filtrage de Kalman (lien entre EKF et UKF) reste vrai, y compris dans un cadre invariant. Les propriétés d'invariance de l'IUKF nouvellement développées sont également observables lorsque l'on compare les erreurs d'estimation commises sur l'état du système observé. Le 
traitement de données simulées nous permet de calculer ces erreurs. De tous les résultats accumulés jusqu'à présent, il ressort que le filtrage de Kalman invariant introduit par JP. Condomines, C. Seren et G. Hattenberger, à l'instar des observateurs invariants déjà existants dans la littérature, l'IUKF permet de caractériser avec précision l'incertitude sur l'état estimé pour certaine trajectoire dite permanente $I=\psi_{\hat{\mathbf{x}}^{-1}}(u)=$ cst. La relative constance de celle-ci en fait une information potentiellement très utile à exploiter dans le cadre d'autres problématiques (commande robuste, FDIR, etc.).
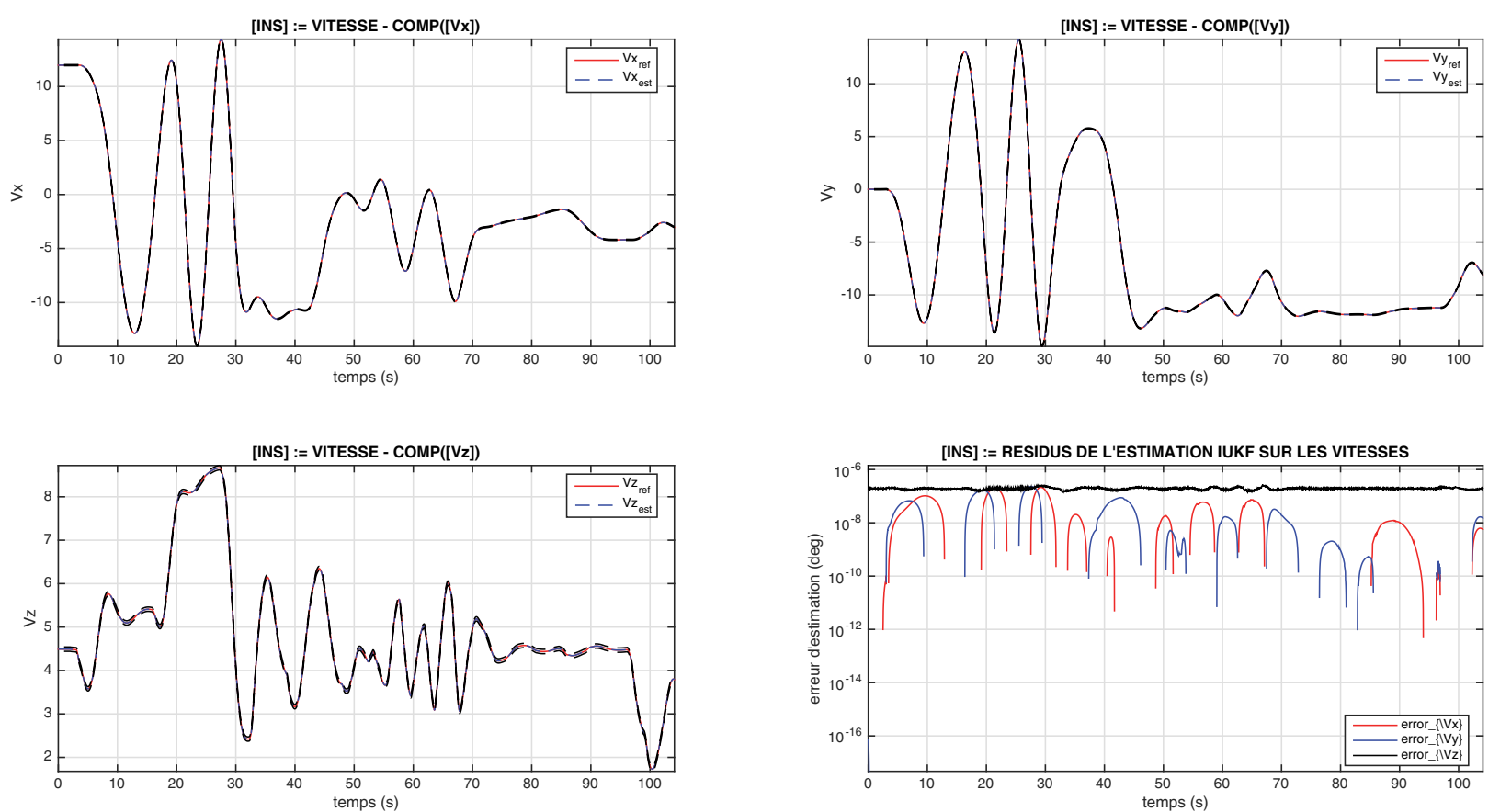

Figure 14. INS-IUKF : estimation des vitesses et erreurs d'estimation
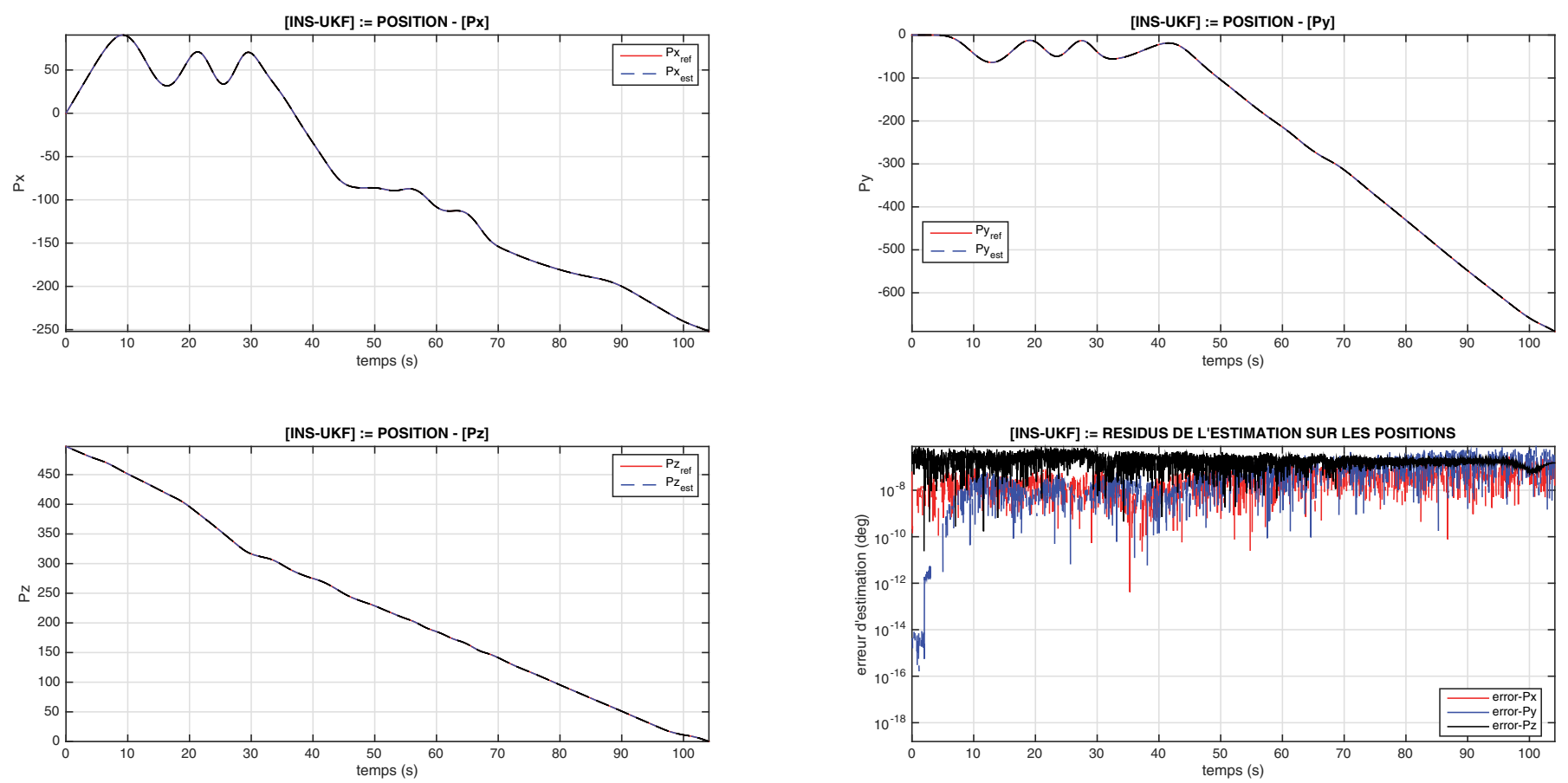

Figure 15. INS-UKF : estimation des positions et erreurs d'estimation 

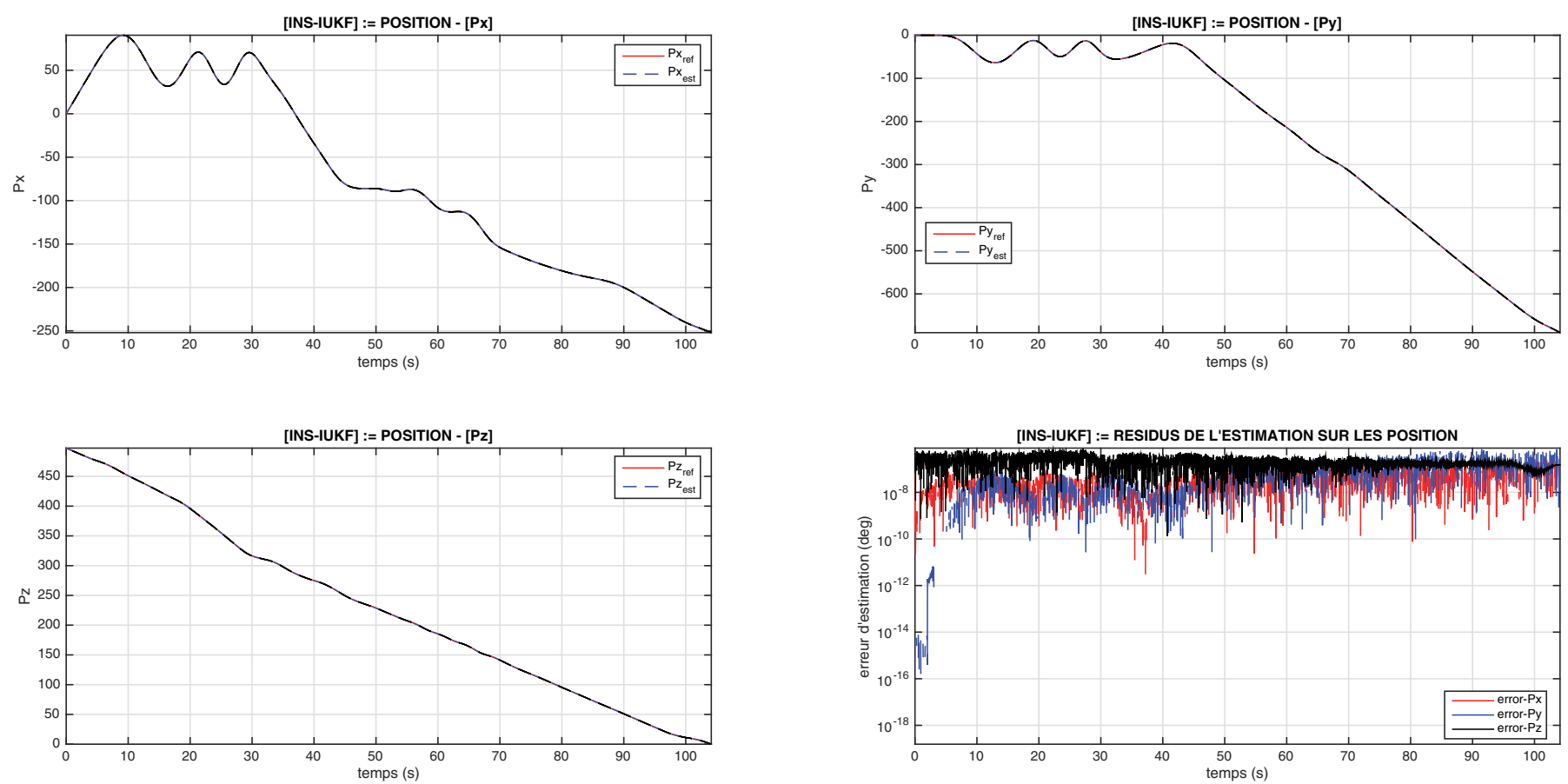

Figure 16. INS-IUKF : estimation des positions et erreurs d'estimation
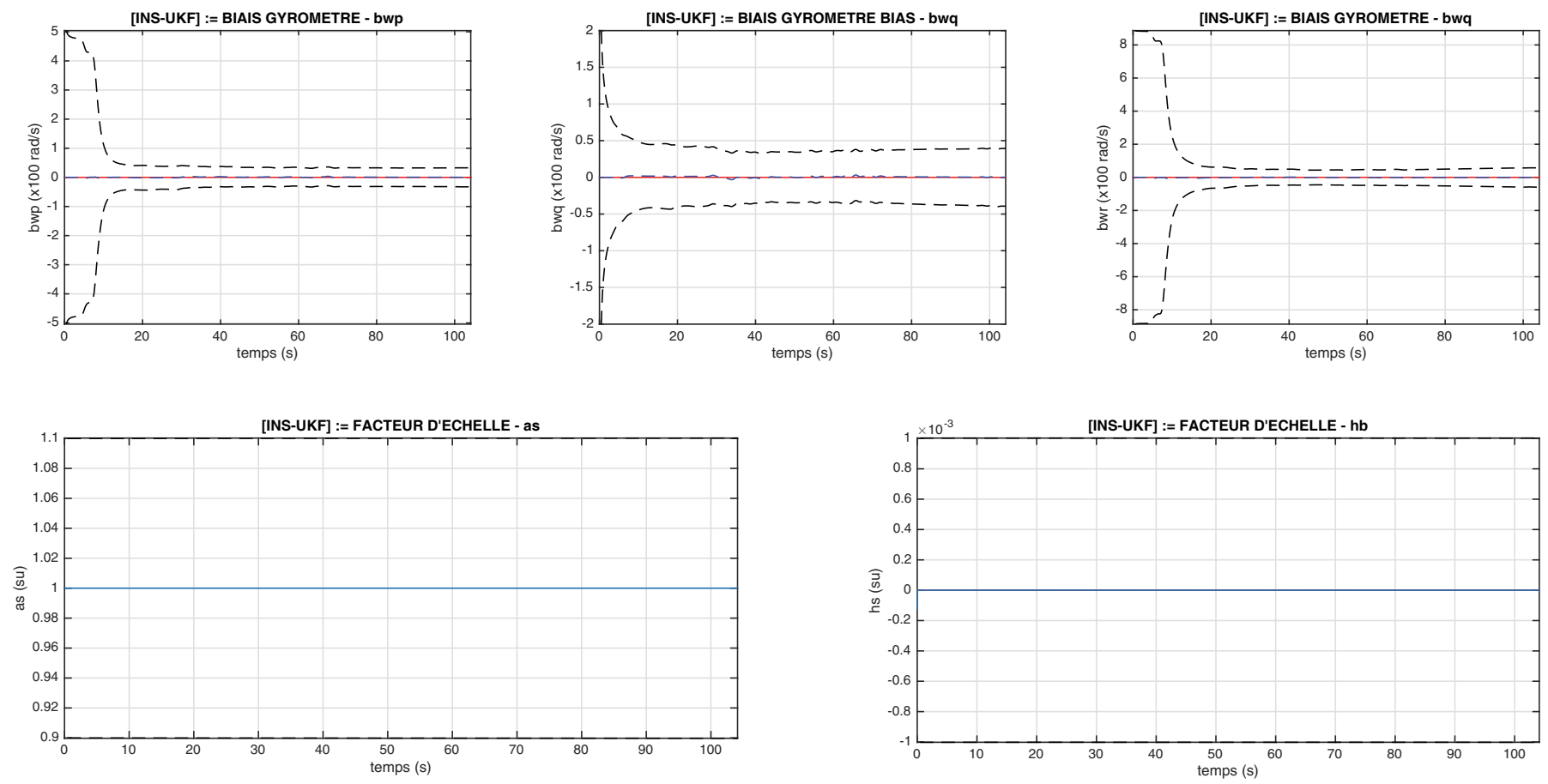

Figure 17. INS-UKF : estimation des imperfections : biais $\omega_{b}$ 

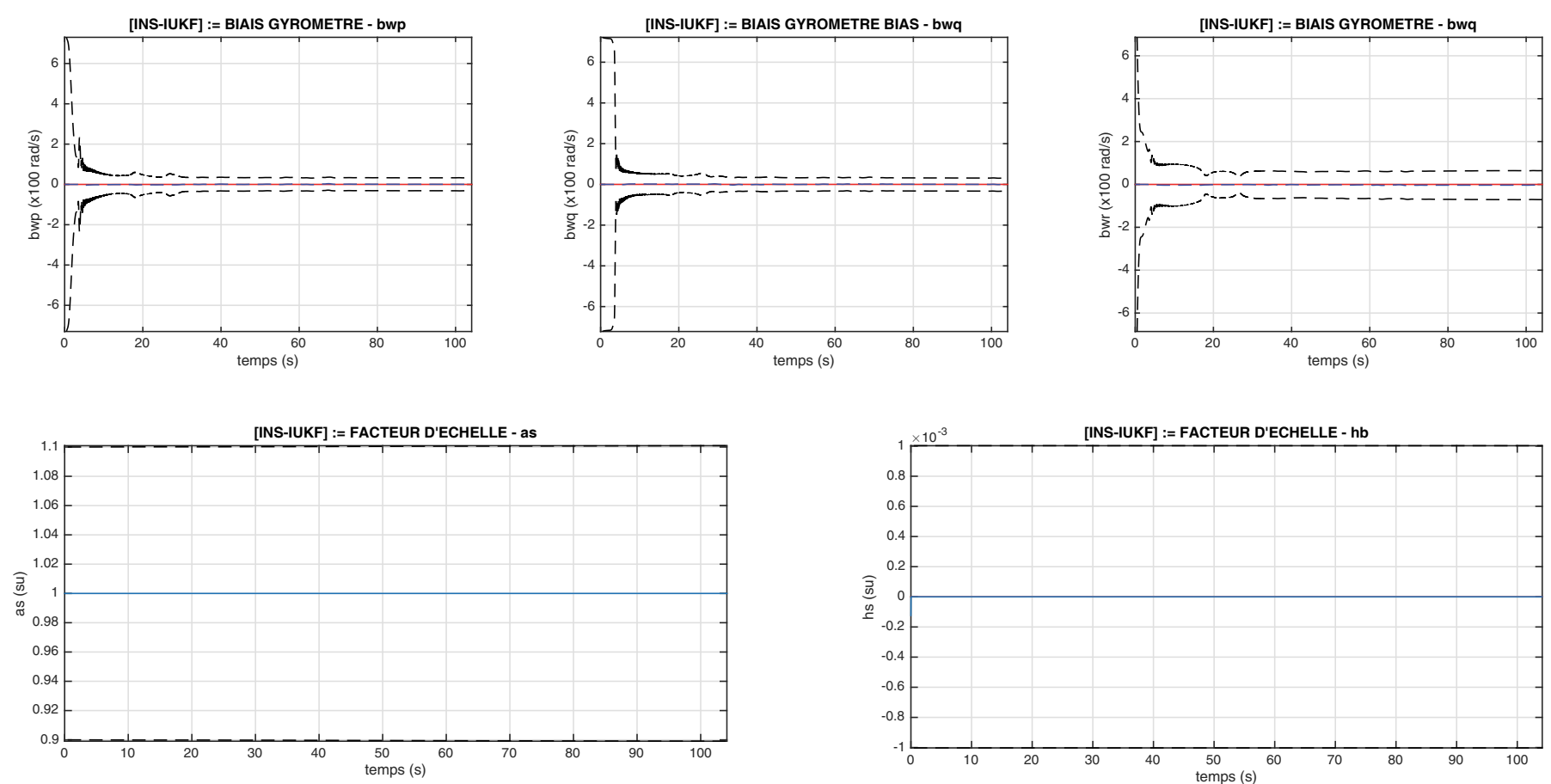

Figure 18. INS-IUKF : estimation des imperfections : biais $\omega_{b}$

\section{Bibliographie}

Aghannan, N., \& Rouchon, P. (2002). On invariant asymptotic observers. In Proceedings of the 41 st IEEE Conference on Decision and Control, Las Vegas (NV), USA, 10-13 December 2002 (pp. 1479-1484).

Barczyk, M., Bonnabel, S., \& Deschaud, J-E. (2015). Invariant EKF Design for Scan Matching-Aided Localization. IEEE Tansaction on Control Systems Technology, 23 (6), 2440-2448.

Barczyk, M., \& Lynch, A. F. (2013). Invariant Observer Design for Helicopter UAV Aided Inertial Navigation System. IEEE Transaction on Control Systems Technology, 21 (3), 791-806.

Barrau, A., \& Bonnabel, S. (2015). Intrinsic filtering on Lie groups with applications to attitude estimation. IEEE Tansaction on Automatic Control, 60 (2), 436-449.

Barrau, A., \& Bonnabel, S. (2014). Invariant particle filtering with application to localization. In Proceedings of the 53rd IEEE Conference on Decision and Control, Los Angeles (CA), USA, 15-17 December 2014 (pp. 5599-5605).

Barczyk, M., Bonnabel, S., \& Deschaud, J-E. (2015). Invariant EKF Design for Scan Matching-Aided Localization. IEEE Tansaction on Control Systems Technology, 23 (6), 2440-2448.

Bonnabel, S., Martin, P., \& Rouchon, P. (2009). Non-linear symmetry-preserving observers on Lie groups. IEEE Tansaction on Automatic Control, 54 (7), 1709-1713.

Bonnabel, S., Martin, P., \& Salaün, E. (2009). Invariant Extended Kalman Filter : Theory and Application to a VelocityAided Attitude Estimation Problem. In Proceedings of the 48th IEEE Conference on Decision and Control, Shanghai, China, 16-18 December 2009 (pp. 1297-1304).

Bonnabel, S., Martin, P., \& Rouchon, P. (2008). Symmetry-preserving observers. IEEE Tansaction on Automatic Control, $53(11), 2514-2526$.

Bonnabel, S. (2007). Left-invariant extended Kalman filter and attitude estimation. In Proceedings of the 46th IEEE Conference on Decision and Control, New Orleans (LA), USA, 12-14 December 2007 (pp. 1027-1032).

Bonnabel, S et al. (2007). An intinsic CramérRao bound on SO(3) for (dynamic) attitude filtering In arXiv :1503.04701v2, 2015.

Bourmaud, G., Mégret,R., Giremus, A., Berthoumieu, Y. (2013). Discrete extended Kalman filter on Lie groups. Signal Processing Conference (EUSIPCO), Proceedings of the 21st European. 
Bourmaud, G., Mégret,R., Giremus, A., Berthoumieu, Y. (2014). Continuous-Discrete Extended Kalman Filter on Matrix Lie Groups Using Concentrated Gaussian Distributions. Journal Math Imaging Vis, DOI 10.1007/s10851-014-0517-0.

Condomines, J-P. (2015). Nonlinear state estimation for guidance and navigation of unmanned aerial vehicles flying in a complex environment. PhD thesis, University of Toulouse, ISAE.

Condomines, J-P., Seren, C., \& Hattenberger, G. (2014). Pi-Invariant Unscented Kalman Filter for Sensor Fusion. In Proceedings of the 53rd IEEE Conference on Decision and Control, Los Angeles (CA), USA, 15-17 December 2014 (pp. 1035-1040).

Condomines, J-P., Seren, C., \& Hattenberger, G. (2013). Nonlinear state estimation using an invariant unscented Kalman filter. In Proceedings of the 2013 AIAA Guidance, Navigation and Control Conference, Boston (MA), USA, 19-2 August 2013 (pp 1-15).

Condomines, J.P., Seren C., \& Hattenberger G. (2017). Optimal Invariant Observers Theory for Nonlinear State Estimation. Multisensor Attitude Estimation Fundamental Concepts and Applications Edited by Fourati Hassen Belkhiat Djamel Eddine Chouaib Iniewski Krzysztof, Taylor \& Francis, pp 391-408, 2016, 978-1-4987-4571-0, ISBN : 978-1-4987-4580-2

Crassidis, J. L., Markley, F. L., \& Cheng, Y. (2007). Survey of Nonlinear Attitude Estimation Methods. Journal of Guidance, Control and Dynamics, 30(1), 12-28.

Mahony, R and Hamel, T and Pflimlin, J-M(2008) Nonlinear complementary filters on the special orthogonal group. IEEE Transaction Automatic Control,53,p1203-1218,2008.

Julier, S. J. (2002). The scaled unscented transformation. In Proceedings of the 2002 American Control Conference, Anchorage (AK), USA, 8-10 May 2002 (pp. 4555-4559).

Khosravian, A., Trumpf, J., Mahony, R., \& Lageman, C. (2015). Observers for invariant systems on Lie groups with biased input measurements and homogeneous outputs. Automatica, 55 (Issue C), 19-26.

Lagemann, C J T and Mahony, R (2008). Observer design for invariant systems with homogeneous observations. In arXiv :0810.0748,2008.

Li, D., Li, Q., Tang, L., Yang, S., Cheng, N., . .Song, J. (2015). Invariant Observer-Based State Estimation for Micro-Aerial Vehicles in GPS-Denied Indoor Environments Using an RGB-D Camera and MEMS Inertial Sensors. Micromachines, 6 , 487-522.

Martin, P., \& Salaün, E. (2008). An invariant observer for earth-velocity-aided attitude heading reference systems. In Proceedings of the 17th IFAC World Congress, Seoul, Korea, 6-11 July 2008 (pp. 9857-9864).

Martin, P., \& Salaün, E. (2007). Invariant observers for attitude and heading estimation from low-cost inertial and magnetic sensors. In Proceedings of the 46th IEEE Conference on Decision and Control, New Orleans (LA), USA, 12-14 December 2007 (pp. 1039-1045).

Maithripala, D and Dayawansa, W P and Berg, J M (2005) Intrinsic observer-based stabilization for simple mechanical systems on Lie Groups. In SIAM Journal on Control and Optimization,44,(pp1691-1711), 2005.

Martin,P., \& Rouchon,P., and J. Rudolph (2004). Invariant tracking. ESAIM : Control, Optimisation and Calculus of Variations , $10: 1-13,2004$.

Sarkka, S. (2007). On unscented Kalman filtering for state estimation of continous-time nonlinear systems. IEEE Transaction on Automatic Control, 52 (9), 1631-1641.

van der Merwe, R., \& Wan, E. A. (2001). The square-root unscented Kalman filter for state and parameter estimation. In Proceedings of the IEEE International Conference on Acoustics, Speech and Signal Processing, Salt Lake City (UT), USA, 7-11 May 2001 (pp. 3461-3464).

Vasconcelos, J and Silvestre, C and Oliveira, (2001). A nonlinear observer for rigid body attitude estimation using vector observations. In 17th World Congres. The International of Automatic Control,p. 8599-8604, 2008. 